\title{
1 Cherry-picking information: humans actively sample 2 evidence to support prior beliefs
}

3

\author{
Paula Kaanders ${ }^{1,2,}$, Pradyumna Sepulveda ${ }^{3}$, Tomas Folke ${ }^{4}$, Pietro Ortoleva ${ }^{5}$, and \\ Benedetto De Martino ${ }^{3,6, *}$ \\ ${ }^{1}$ Department of Experimental Psychology, University of Oxford, Oxford, United Kingdom; \\ ${ }^{2}$ Wellcome Centre for Integrative Neuroimaging, University of Oxford, Oxford, United \\ Kingdom; ${ }^{3}$ Institute of Cognitive Neuroscience, University College London, London, \\ United Kingdom; ${ }^{4}$ Centre for Business Research, Cambridge Judge Business School, \\ University of Cambridge, Cambridge, United Kingdom; ${ }^{5}$ Department of Economics, \\ Columbia University, New York, United States of America; ${ }^{6}$ Wellcome Centre for Human \\ Neuroimaging, University College London, London, United Kingdom \\ *Correspondence: paula.kaanders@psy.ox.ac.uk, benedettodemartino@gmail.com
}

\section{$\underline{\text { Abstract }}$}

No one likes to be wrong. Previous research has shown that participants may underweight information incompatible with previous choices, a phenomenon called confirmation bias. In this paper we argue that a similar bias exists in the way information is actively sought. We investigate how choice influences information gathering using a perceptual choice task and find that participants sample more information from a previously chosen alternative. Furthermore, the higher the confidence in the initial choice, the more biased information sampling becomes. As a consequence, when faced with the possibility of revising an earlier decision, participants are more likely to stick with their original choice, even when incorrect. Critically, we show that agency controls this phenomenon. The effect disappears in a fixed sampling condition where presentation of evidence is controlled by the experimenter, suggesting that the way in which confirmatory evidence is acquired critically impacts the decision process. These results suggest active information acquisition plays a critical role in the propagation of strongly held beliefs over time. 
We are constantly deciding what information to sample to guide future decisions. This is no easy feat, as there is a vast amount of information available in the world. In theory, an agent should look for information to maximally reduce their uncertainty (Schulz \& Gershman, 2019; Schwartenbeck et al., 2019; Yang et al., 2016). There is some evidence that this is indeed what humans do (Kobayashi \& Hsu, 2019; Meyer \& Shi, 1995; Steyvers et al., 2009; Wilson et al., 2014), but a range of other drivers of information search have been identified that make us deviate from the sampling behaviour of optimal agents (Eliaz \& Schotter, 2007; Gesiarz et al., 2019; Hunt et al., 2016; Kobayashi et al., 2019; Rodriguez Cabrero et al., 2019; Sharot, 2011; van Lieshout et al., 2018; Wang \& Hayden, 2019).

Confirmation bias is defined as the tendency of agents to seek out or overweight evidence that aligns with their beliefs while avoiding or underweighting evidence that contradicts them (Hart et al., 2009; Lord et al., 1979; Nickerson, 1998; Stroud, 2008; Wason, 1960, 1968). This effect has often been described in the context of what media people choose to consume (Bakshy et al., 2015; Bennett \& Iyengar, 2008; Pariser, 2012). Recently, cognitive scientists have realised confirmation bias may not be restricted to this domain and may reveal a fundamental property of the way in which the brain drives information search. As such, researchers have started to investigate confirmation bias in simple perceptual and value-based choice tasks. For example, sensitivity to new information has been shown to decrease after choice (Bronfman et al., 2015), while Talluri, Urai et al. (2018) have shown that sensitivity to a stimulus is increased when it is consistent with the stimulus presented before. Furthermore, in Palminteri et al. (2017) learning rates were found to be higher for feedback indicating positive obtained outcomes as well as for negative forgone outcomes. This effect has been replicated in recent studies that suggest confirmation bias during learning might be adaptive in some contexts (Tarantola, Folke et al., 2021; Lefebvre et al., 2020; Salem-Garcia et al., 2021).

Most of the existing research on perceptual decision-making has focused on the weighting of incoming information during evidence accumulation or belief updating. Other findings suggest that agents also actively search for confirmatory evidence (Hunt et al., 2016, 2018; Tolcott et al., 1989; Wason, 1968), but none of these experiments investigated the effect of biased information sampling in simple, perceptual choice. As most paradigms currently used to study confirmation bias do not explicitly focus on active information sampling performed by the agent, therefore it is unclear whether confirmation bias arises from evidence underweighting, biased information sampling, or both.

Another important aspect of confirmation bias is how it modulates and is modulated by decision confidence. Confidence is known to play an important role in guiding decisions (Bogacz et al., 2010; Boldt et al., 2019; Folke et al., 2017; Rabbitt, 1966; Yeung \& Summerfield, 2012). It is therefore likely it may also guide the information search preceding choice (Desender et al., 2019). In fact, Rollwage et al. (2020) recently showed that confidence indeed affects a neural signal of confirmatory evidence integration. By definition, the higher confidence is in a choice, the stronger the decision-maker's belief is in the correctness of this choice (Fleming \& Lau, 2014; Pouget et al., 2016). Accordingly, we predict that confirmation bias in information sampling will be stronger after choices made with high confidence. 
89 We used two perceptual binary forced choice tasks with two choice phases separated by 90 a free sampling phase to test our hypotheses that confirmation bias arises from biased 91 information sampling, that this effect influences future choice and that confidence

92

93

94

95

96

97

98

99

100

101

102

103

104

105

106

107

108

109

110

111

112

113

114

115

116

117

118

119

120

121

122

123

124

125

126

127

128 mediates this behavioural tendency.

\section{Results}

Here we present the results of two independent experiments we conducted to test our hypotheses.

\section{Experiment 1}

Participants performed a perceptual two-alternative forced choice (2AFC) task (Figure $1 \mathrm{~A})$, in which they were briefly presented with patches containing multiple dots. Participants were asked to judge which patch contained the most dots. After their initial choice (phase 1), they rated their confidence that they were correct. Subsequently, in the second phase of the trial (phase 2) participants could freely sample (i.e., see) each dot patch using key presses to switch between them as frequently as they liked for $4000 \mathrm{~ms}$. Participants could only view one patch at a time. They were then prompted to make a second choice (phase 3), in which they could either confirm their initial choice or change their mind. After this second decision, they again reported their degree of confidence.

As expected, participants' performance was highly sensitive to the amount of perceptual evidence presented in a given trial (i.e. the difference in the amount of dots in the left and right patches; Fig 1B). Participants were also more accurate in the second choice compared to the first choice, showing that they used the additional sampling time between choice phases to accumulate additional perceptual evidence that allowed them to increase accuracy on the second choice $\left(\mathrm{t}_{27}=8.74, \mathrm{p}<0.001\right)$.

Our main hypothesis was that participants would prefer to gather perceptual information that was likely to confirm their initial decision. Conversely, we expected them to be less likely to acquire evidence that would disconfirm it. To perform well on this task, an agent would have to attend equally to the two patches, as the goal requires computing the difference in dots between the two patches. Therefore, any imbalance in sampling time would not necessarily be beneficial to completing the task. However, we expected that in the free sampling phase (phase 2) of the trial participants would spend more time viewing their chosen patch. In line with this hypothesis, during the sampling phase, participants spent more time viewing the patch they chose in the first decision phase relative to the unchosen alternative (Figure $1 \mathrm{C}$; $\mathrm{t}_{27}=7.28, \mathrm{p}<0.001$ ). 
bioRxiv preprint doi: https://doi.org/10.1101/2021.06.29.450332; this version posted June 30, 2021. The copyright holder for this preprint

(which was not certified by peer review) is the author/funder, who has granted bioRxiv a license to display the preprint in perpetuity. It is made available under aCC-BY-NC-ND 4.0 International license.

\section{- Experiment 1 -}

A
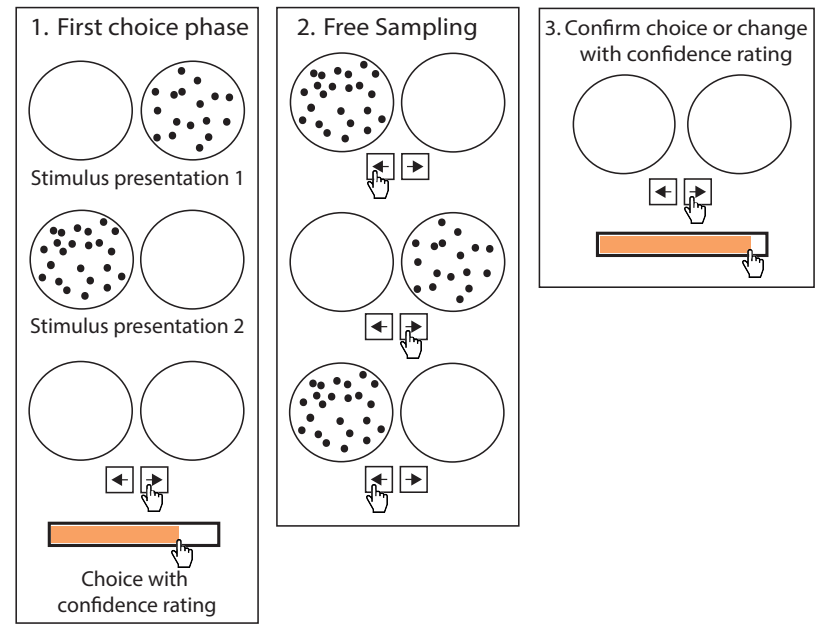

B
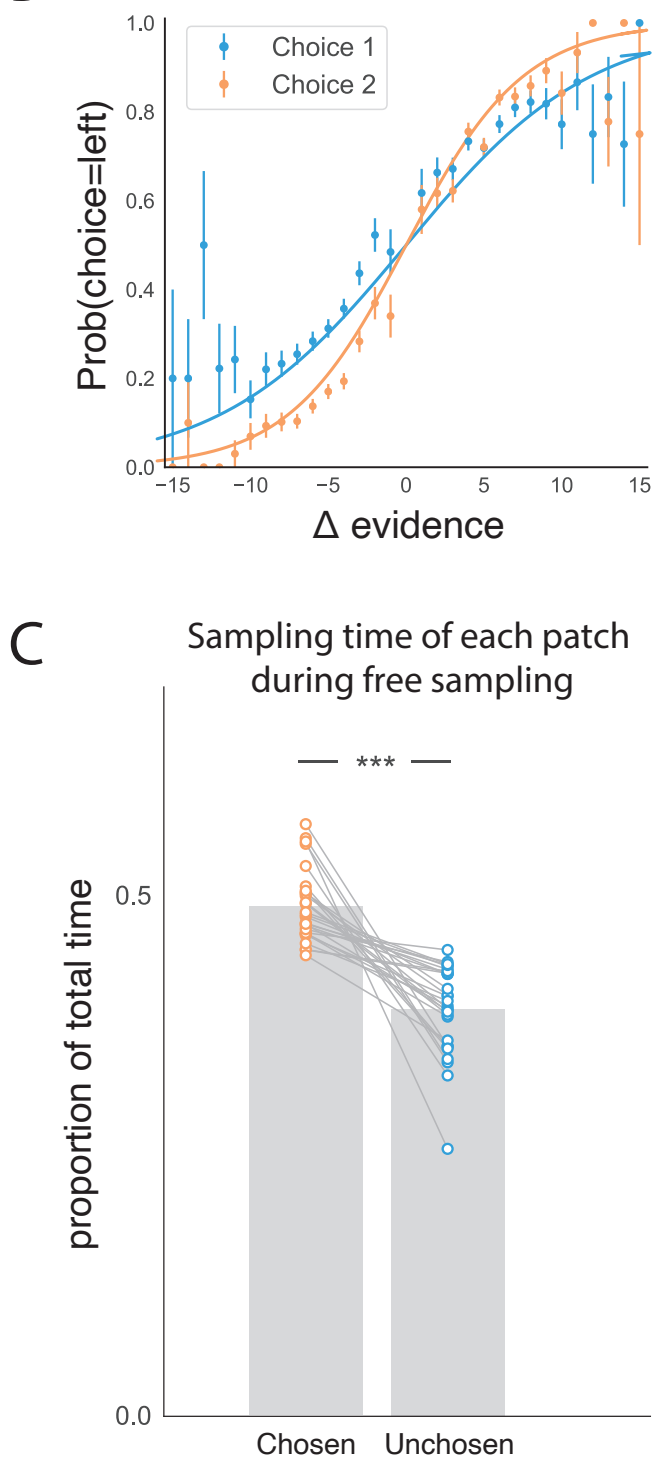
Figure 1. Task design and participant behaviour for experiment 1. (A) Task structure. Participants had to choose which of two dot patches contained the most dots (phase 1) and rate their confidence in this choice. Then participants were given $4000 \mathrm{~ms}$ to view the dots which they could allocate between the two patches in whichever way they liked (phase 2) by pressing the left and right arrow keys. Finally, participants made a second choice about the same set of stimuli and rated their confidence again (phase 3). (B) Participants effectively used the stimuli to make correct choices and improved upon their performance on the second choice. This psychometric curve is plotting the probability of choosing the left option as a function of the evidence difference between the two stimuli for each of the two choice phases. (C) In the free sampling phase (phase 2) participants spent more time viewing the stimulus they chose on the preceding choice phase than the unchosen option. Data points represent individual participants.

141

142

143

144

145

146

147

148

149

150

151

152

153

154

155

156

157

158

Confidence in a choice reflects the strength of the participant's belief that their choice was correct. Consequently, we hypothesised that participants' preference for gathering confirmatory evidence for a given choice would be modulated by their confidence in that choice. As such, we expected choices made with higher confidence would lead to a stronger sampling bias favouring the chosen patch over the unchosen patch. In a hierarchical regression predicting sampling time difference between the two patches, we found a significant interaction between choice and confidence, such that the higher the degree of confidence was in the first choice, the more sampling was biased in favour of that choice (Figure 2; $\mathrm{t}_{26.96}=5.26, \mathrm{p}<0.001$; see supplemental materials $\mathrm{S} 1$ ). We also saw a significant main positive effect of choice on sampling time difference, such that a chosen stimulus was likely to be sampled for longer during the sampling phase (Figure $2 \mathrm{~B} ; \mathrm{t}_{26.96}=9.64, \mathrm{p}<0.001$ ) as shown in the previous section. There was also a significant main positive effect of evidence difference on sampling time difference, whereby the correct stimulus (containing the most dots) was sampled for longer (Figures 2B; $\left.t_{26.96}=13.02, p<0.001\right)$.

\section{- Experiment 1 -}

A

Increased confidence leads to more bias

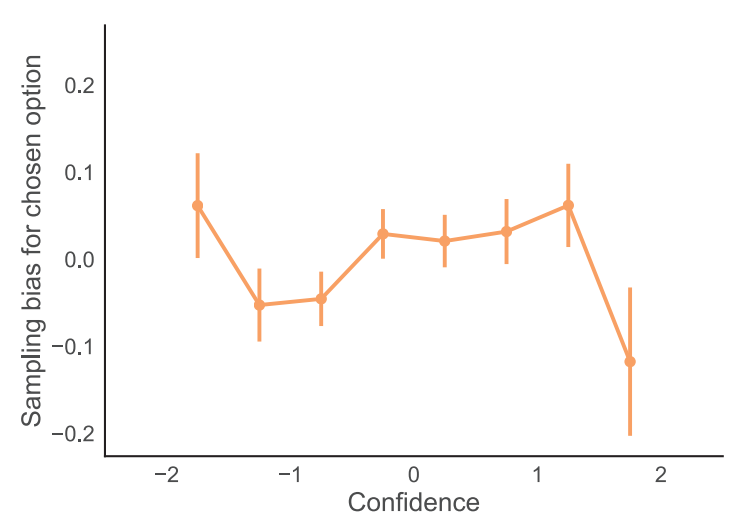

B

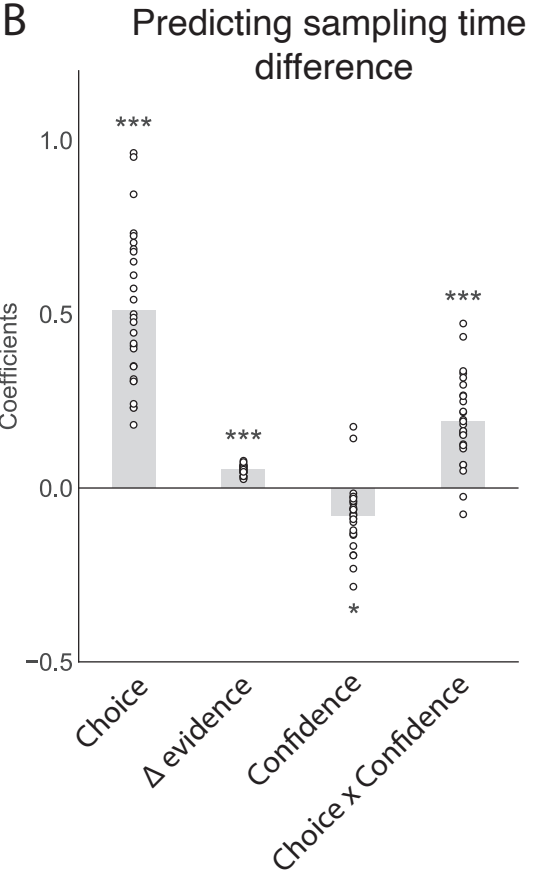

C Predicting change of mind

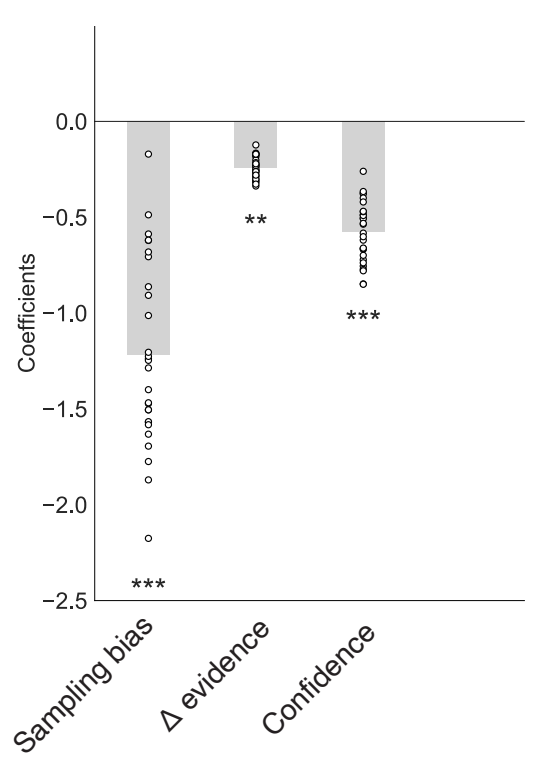

Figure 2. The effect of choice on sampling behaviour is mediated by confidence in experiment 1 . Participants were less likely to change their mind if they showed a strong sampling bias for their initially chosen option in the sampling phase. (A) Sampling bias in favour of the chosen option increases as a 
function of confidence in the initial choice. Confidence and sampling bias are both normalised in this plot. (B) There is a significant main effect of choice on sampling time difference, such that an option is sampled for longer if it was chosen, and a significant interaction effect of Choice x Confidence, such that options chosen with high confidence are sampled for even longer. (C) There is a main negative effect of sampling bias on change of mind, such that participants were less likely to change their mind in the second decision phase (phase 3) the more they sampled their initially chosen option in the free sampling phase (phase 2). (B-C) Plotted are fixed-effect coefficients from hierarchical regression models predicting the sampling time (how long each patch was viewed in the sampling phase) difference between the left and right stimuli. Data points represent regression coefficients for each individual participant.

Our choices are determined by the evidence we have gathered before making a decision. Therefore, we expected that biased sampling in the free sampling phase (phase 2) would impact decisions in the second decision phase (phase 3). Specifically, we hypothesised that the more strongly participants preferred sampling their chosen patch, the more likely they were to choose it again. Using a multinomial hierarchical logistic regression, we found that bias in sampling time towards the previously chosen option was indeed predictive of future choice. In other words, the more participants sampled a previously chosen option, the more likely they were to choose it again (Figure $2 \mathrm{C}$; $\mathrm{z}=-11.0, \mathrm{p}<0.001$; see S2). Furthermore, evidence difference and confidence were significantly negatively related to subsequent changes of mind, whereby participants were less likely to change their mind if their initial choice was correct and if it was made with high confidence (Figure 2C; main effect of evidence difference $-\mathrm{z}=-3.06, \mathrm{p}<0.01$, main effect of confidence $-\mathrm{z}=-10.12, \mathrm{p}<0.001)$.

\section{Experiment 2}

While the results from the first experiment showed an effect of biased sampling on subsequent choice, it was not clear whether this effect arises from biased evidence accumulation caused by differential exposure to the perceptual evidence, or if the sampling choices themselves drive the effect. In other words, would the same choice bias appear if participants were passive recipients of biased sampling, or does the choice bias require that participants make their own sampling decisions?

We addressed this question in a follow-up eye-tracking study (Figure 3A) in which we introduced a control task, a 'fixed-viewing condition', in which participants did the same task, but did not have the possibility to freely sample the patches in phase 2 . Instead, the dot patches were shown for a set amount of time. In one-third of trials, the patches were shown an equal amount of time; in two-thirds of trials, one patch was shown three times longer than the other. Each participant completed two sessions, one session involved free sampling, similar to Experiment 1 . The other involved a fixed-viewing control task. Session order was pseudo-randomized between participants. Furthermore, presentation of the visual stimuli and all the participants' responses were gaze-contingent. This meant that during the sampling phase (phase 2), the dot patches were only presented when participants fixated inside the patch. Furthermore, we hypothesised that sampling bias might be stronger when more time to sample is available. Specifically, we expected that participants might become more biased in their sampling throughout the sampling phase as they gradually become more confident in their belief. Therefore, we manipulated the length of phase 2 to be either $3000 \mathrm{~ms}, 5000 \mathrm{~ms}$, or $7000 \mathrm{~ms}$. Again, participants were sensitive to the difficulty of the given trials (Figure 3B) and were more accurate on the 
214 second choice compared to the first choice $\left(\mathrm{t}_{17}=6.80, \mathrm{p}<0.001\right)$.

216 We also replicated our main result in the free sampling condition showing that 217 participants spent more time viewing the patch they just chose (Figure $3 C ; t_{17}=3.52$, $218 \mathrm{p}<0.01)$. Furthermore, the size of this sampling time bias was proportional to the total 219 amount of sampling time available in study 2 (Figure S3.1), suggesting that there was no 220 particular period of time within the sampling phase where confirmatory information 221 sampling was more likely contrary to our expectation. 
bioRxiv preprint doi: https://doi.org/10.1101/2021.06.29.450332; this version posted June 30, 2021. The copyright holder for this preprint (which was not certified by peer review) is the author/funder, who has granted bioRxiv a license to display the preprint in perpetuity. It is made available under aCC-BY-NC-ND 4.0 International license.

A
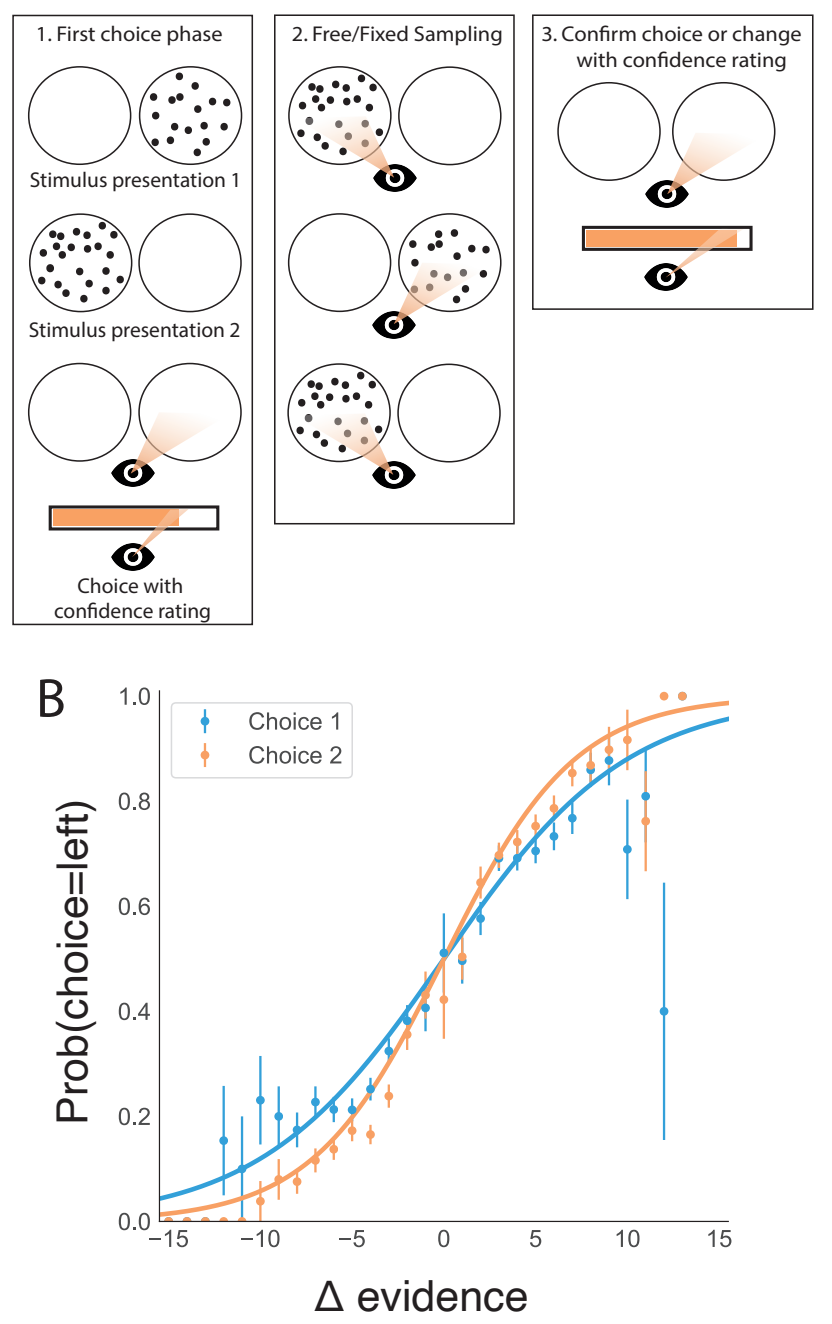

C

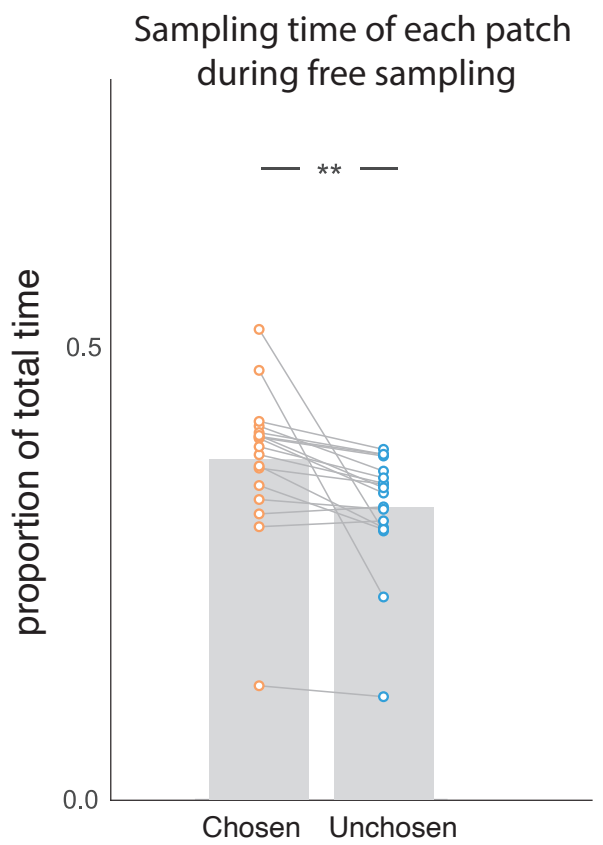


bioRxiv preprint doi: https://doi.org/10.1101/2021.06.29.450332; this version posted June 30, 2021. The copyright holder for this preprint (which was not certified by peer review) is the author/funder, who has granted bioRxiv a license to display the preprint in perpetuity. It is made available under aCC-BY-NC-ND 4.0 International license.

224 Figure 3. Task design and participant behaviour for experiment 2. (A) Task structure. Participants had to choose which of two dot patches contained the most dots (phase 1) and rate their confidence in this choice. Then participants were given $3000 \mathrm{~ms}, 5000 \mathrm{~ms}$, or $7000 \mathrm{~ms}$ to view the dots which they could allocate between the two patches in whichever way they liked (phase 2) by looking inside the circles. Finally, participants made a second choice about the same set of stimuli and rated their confidence again (phase 3). (B) Participants effectively used the stimuli to make correct choices and improved upon their performance on the second choice. This psychometric curve is plotting the probability of choosing the left option as a function of the evidence difference between the two stimuli for each of the two choice phases. (C) In the free sampling condition during the sampling phase (phase 2) participants spent more time viewing the stimulus they chose on the preceding choice phase than the unchosen option. Data points represent 
237 This new experiment also replicated the mediating effect of confidence on how 238 sampling bias affects subsequent choice. We again found a significant interaction 239 between choice and confidence (Figure 4A-B; $t_{16.97}=4.29, \mathrm{p}<0.001$; see supplemental 240 materials S1) and replicated the main positive effects of choice and evidence difference 241 on sampling time difference (Figure 4B; main effect of choice: $\mathrm{t}_{16.97}=2.90, \mathrm{p}<0.01$; main 242 effect of evidence difference: $\left.\mathrm{t}_{16.97}=9.21, \mathrm{p}<0.001\right)$.

\section{- Experiment 2 -}

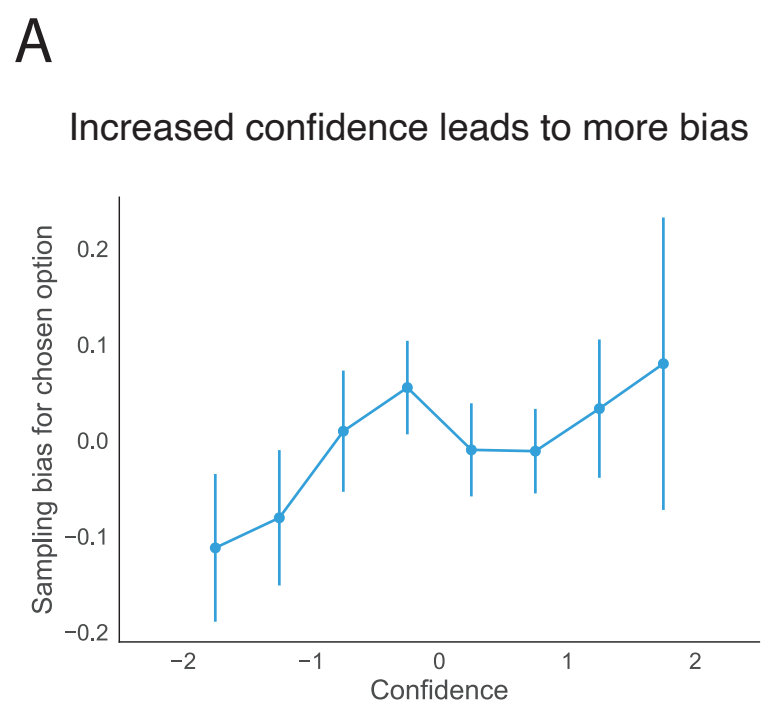

C

Predicting change of mind

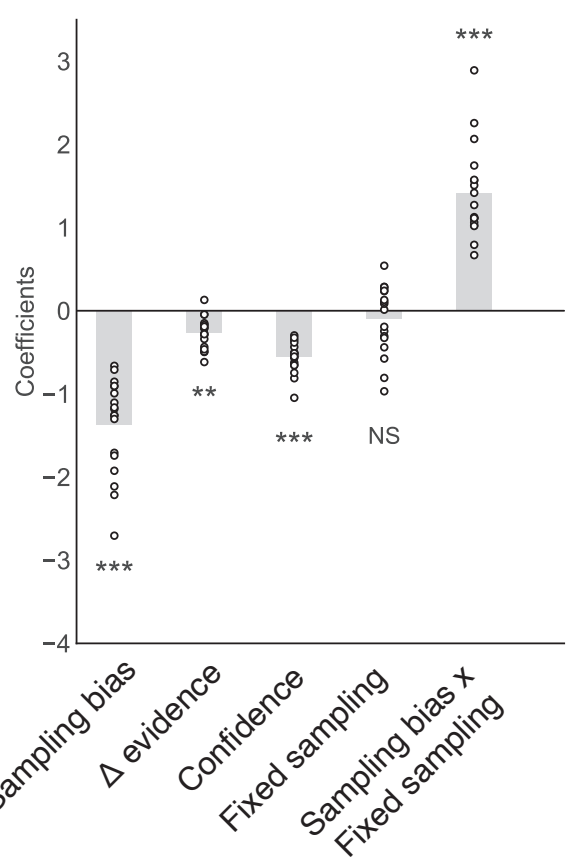

B Predicting sampling time difference

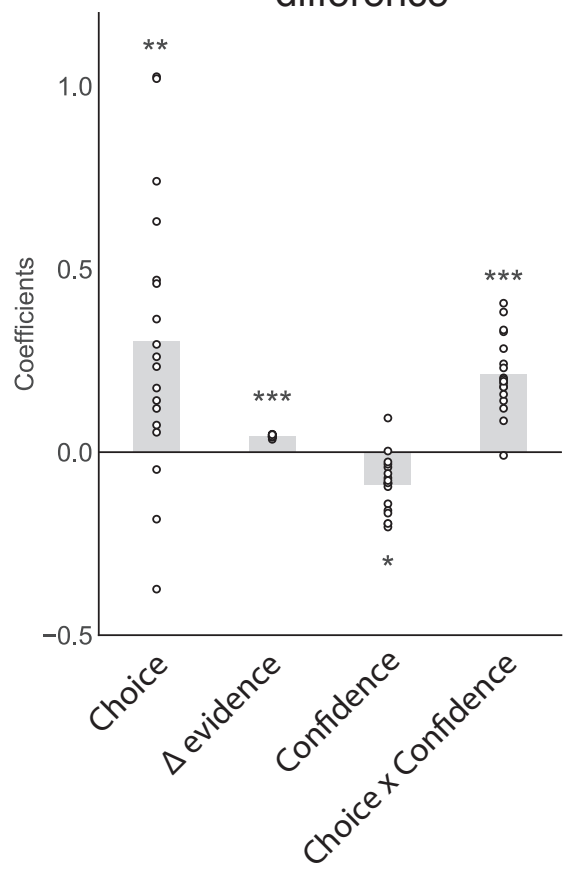

D Sampling bias only affects choice in free sampling trials

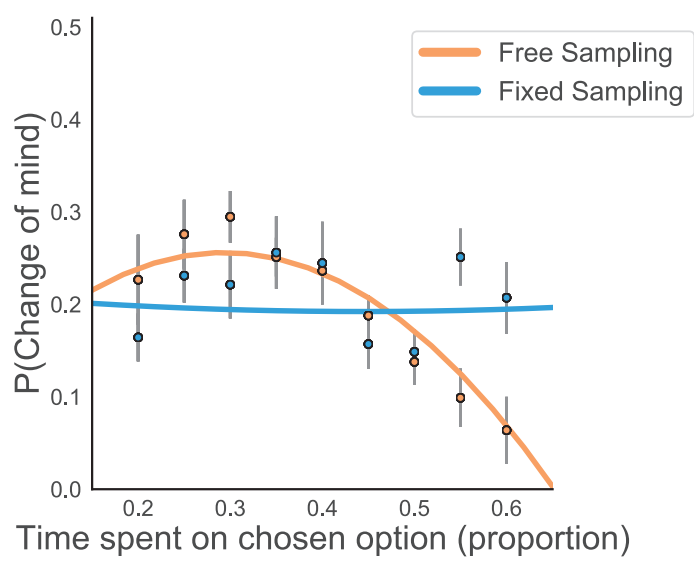


Figure 4. The effect of choice on sampling behaviour is mediated by confidence in experiment 2 . Participants were less likely to change their mind if they showed a strong sampling bias for their initially chosen option in the sampling phase, but this was only the case in the free sampling condition. (A) Sampling bias in favour of the chosen option increases as a function of confidence in the initial choice. Confidence and sampling bias are both normalized in this plot. (B) There is a significant main effect of choice on sampling time difference, such that an option is sampled for longer if it was chosen, and a significant interaction effect of Choice $\mathrm{x}$ Confidence, such that options chosen with high confidence are sampled for even longer. (C) There is a main negative effect of sampling bias on change of mind, such that participants were less likely to change their mind in the second decision phase (phase 3) the more they sampled their initially chosen option in the free sampling phase (phase 2). The main effect of sampling bias on change of mind disappears in the fixed sampling condition, which can be seen by the positive interaction term Sampling bias $\mathrm{x}$ Fixed sampling which entirely offsets the main effect. The analysis includes a dummy variable 'Fixed Sampling' coding whether the trial was in the fixed-viewing condition. (B-C) Plotted are fixed-effect coefficients from hierarchical regression models predicting the sampling time (how long each patch was viewed in the sampling phase) difference between the left and right stimuli. Data points represent regression coefficients for each individual participant. (D) The probability that participants change their mind on the second choice phase is more likely if they looked more at the unchosen option during the sampling phase. The plot shows the probability that participants changed their mind as a function of the time spent sampling the initially chosen option during phase 2 . The lines are polynomial fits to the data, while the data points indicate the frequency of changes of mind binned by sampling bias. Note that actual gaze time of the participants is plotted here for both task conditions. The same pattern can be seen when instead plotting the fixed presentation times of the stimuli for the fixed task condition (see Figure S4.1).

Similarly, we replicated the negative effect of sampling bias on subsequent change of mind (Figure 4C; $\mathrm{z}=-7.07, \mathrm{p}<0.001$; see S1) as well as the main negative effects of evidence difference and confidence on change of mind (Figure 4C; main effect of evidence difference: $\mathrm{z}=-2.71, \mathrm{p}<0.01$; main effect of confidence: $\mathrm{z}=-8.86, \mathrm{p}<0.001$ ).

Once we confirmed all the main findings from the first experiment using this new setup, we were able to test our main hypothesis: does the effect of sampling bias on choice we identified require the participant to actively choose which information to sample? In other words, is the effect of confirmation bias on subsequent choice present when confirmatory evidence is passively presented to participants or does it require that confirmatory evidence is actively sought by the decision-maker? In the first case, we would expect to see the same effect in the fixed-viewing condition (in which asymmetric information is provided by the experimenter) as in the free-sampling condition. In the second case, we would expect that the effect of biased information sampling on the subsequent choice disappears in the fixed-viewing condition.

In line with the second prediction, we found that in the fixed-viewing condition, contrary to the free-sampling condition in this experiment and in the first experiment, the amount of time spent viewing the patches in the sampling phase did not significantly affect subsequent choice. In a multinomial hierarchical logistic regression predicting change of mind from the first choice phase to the second choice phase within a given trial, the main effect of sampling bias on change of mind was completely offset by the positive effect of the interaction term between sampling bias and a dummy variable that was set to 1 if a trial was in the fixed-viewing condition (Figures 4C-D; $z=7.24, p<0.001$; see supplemental materials S2). This means that there was no effect of sampling bias on change of mind in the fixed-viewing condition. To check that participants were engaging in this version of 
sampling phase was similar between the two tasks. We found that the number of saccades was actually higher in the fixed-viewing condition than in the main experiment $\left(\mathrm{t}_{17}=-4.22\right.$, $\mathrm{p}<0.001$ ), which means participants were indeed exploring the information in this condition. This confirms that active information sampling by the participant is crucial to the effect of confirmation bias in sampling on future choices.

To further investigate how attention, when freely allocated, shapes the accumulation of evidence and choice bias, we modelled the data from both viewing conditions using the Gaze-weighted Linear Accumulator Model (GLAM; Molter et al., 2019; Sepulveda et al., 2020; Thomas et al., 2019). GLAM belongs to the family of race models with an additional modulation by visual attention (Figure $5 \mathrm{~A}$ ). It is an approximation of a widely used class of models - the attentional Drift Diffusion Model (aDDM; Krajbich et al., 2010; Krajbich and Rangel, 2011) in which the full dynamic sequence of fixations is replaced by the percentage of time spent fixating the choice alternatives. Even-numbered trials were used to fit the model while odd-numbered trials were used to test it. See the Methods section for further details.

GLAM is defined by four free parameters: $\nu$ (drift term), $\gamma$ (gaze bias), $\tau$ (evidence scaling), and $\sigma$ (normally distributed noise standard deviation). The model correctly captured the reaction times (RT) and choice behaviour of the participants at group-level both in the free-sampling (Figure 5B) and fixed-viewing conditions (Figure 5C). More specifically, we found that the model predicted faster RTs when trial difficulty was low ( $\mid \Delta$ Dots| is high; Figures 5B-C, top left). The model also reproduced overall choice behaviour as a function of the number of dots in the patches $\left(\Delta\right.$ Dots $=$ Dots $_{\text {Left }}-$ Dots Right $)$ in both conditions (Figures $5 B-C$, top right). Furthermore, we found gaze allocation $\left(\Delta\right.$ Gaze $\left.=g_{\text {Left }}-g_{\text {Right }}\right)$ predicted the probability of choosing the correct patch in the free-sampling condition (Figure 5C, bottom left). However, to properly test how predictive gaze allocation is of choice, we must account for the effect of evidence ( $\Delta$ Dots) on choice. As such, we used the gaze influence (GI) measure (Thomas et al., 2019), which reflects the effect of gaze on choice after accounting for the effect of evidence on choice. GI is calculated taking the actual choice ( 0 or 1 for right or left choice, respectively) and subtracting the probability of choosing the left item as predicted by a logistic regression with $\Delta$ Dots as a predictor estimated from behaviour. The averaged residual choice probability reflects GI. We found GI estimated purely from participant's behaviour was higher in the free-sampling than in the fixed-viewing condition (comparing average GI by participant, free-sampling condition: Mean $=0.148, \mathrm{SD}=0.169$; fixed-viewing condition: Mean $=0.025, \mathrm{SD}=0.11$; $t_{17}=2.202, p<0.05$ ). This suggests the effect of visual attention on choice was higher in the free-sampling condition. In line with this, the model also predicted a higher GI on corrected choice probability in the free-sampling condition (comparing average GI by individual model predictions, free-sampling condition: Mean $=0.112, \mathrm{SD}=0.106$; fixedviewing condition: Mean $=0.041, \mathrm{SD}=0.055 ; \mathrm{t}_{17}=2.205, \mathrm{p}<0.05$; Figures $5 \mathrm{~B}-\mathrm{C}$, bottom right).

We then tested whether attention affected information integration more when information was actively sought (i.e. the free-sampling condition) compared to when information was given to the participants (i.e. the fixed-viewing condition). We compared 
344 the parameters obtained from the individual fit in the free-sampling and fixed-viewing

345 conditions (Figure 5D). We found a significant variation in the gaze bias parameter (Mean

$346 \gamma_{\text {Free }}=0.81$, Mean $\gamma_{\text {Fixed }}=0.96, \mathrm{t}_{17}=-3.268 ; \mathrm{p}<0.01$ ), indicating a higher influence of gaze

347 on choice in the free-sampling condition. Note that during the fixed-viewing condition,

348 the parameter $\gamma \approx 1$ indicates that almost no gaze bias was present on those trials.

349 Conversely, there was no significant difference for the other parameters between the

350 conditions (Mean $\tau_{\text {Free }}=1.44$, $\tau_{\text {Fixed }}=1.11, \mathrm{t}_{17}=1.17 ; \mathrm{p}=0.25$, n.s.; Mean $\sigma_{\text {Free }}=0.0077$, Mean

$351 \sigma_{\text {Fixed }}=0.0076, \mathrm{t}_{17}=0.128 ; \mathrm{p}=0.89$, n.s.; $v_{\text {Free }}=8.07 \times 10^{-5}, v_{\text {Fixed }}=8.64 \times 10^{-5}, \mathrm{t}_{17}=-1.135 ; \mathrm{p}=$

3520.27 , n.s.). These results suggest that gathering information actively (i.e. free-sampling

353 condition) does not affect the overall speed at which information is integrated, but it

354 specifically modulates the likelihood of choosing the gazed-at option. Finally, to test that

355 the identified effect did not depend on a less variable gaze time range we resampled the

356 data from the free-sampling condition to match the gaze time range in the fixed-viewing

357 condition, and fitted the GLAM again. We replicated our finding even when the gaze time

358 range in the free-sampling condition was reduced to match that in the fixed-viewing

359 condition (Figure S5.1). For more in-depth analyses including model comparisons please

360 see the supplemental materials (S5).

362 Finally, we explored the idea that a sampling bias could arise from the use of previous 363 choice as evidence in its own right, in addition to previously acquired information. 364 Formalizing this idea, we developed an economic model that makes behavioural 365 predictions aligned with our findings (S6). In this model, after the first choice, the prior 366 that feeds into the subsequent active sampling phase is not the belief after the first 367 sampling phase, but rather it is a convex combination of this with the belief that the 368 individuals would hold if they only knew of the previous choice. Subsequent active 369 sampling then varies as a strictly increasing function of this prior. As a result, a sampling 370 bias in favour of the initially chosen option arises, and this bias varies as a function of 371 confidence in the initial choice, as seen in our data. This economic model provides a 372 formal description of the behaviour we observed. At the same time, it suggests that 373 seeking for confirmatory evidence might not arise from a simple low-level heuristic, but 374 is rooted in the way information is acquired and processed (for more details, see S6). 
bioRxiv preprint doi: https://doi.org/10.1101/2021.06.29.450332; this version posted June 30, 2021. The copyright holder for this preprint (which was not certified by peer review) is the author/funder, who has granted bioRxiv a license to display the preprint in perpetuity. It is made available under aCC-BY-NC-ND 4.0 International license.

\section{A}
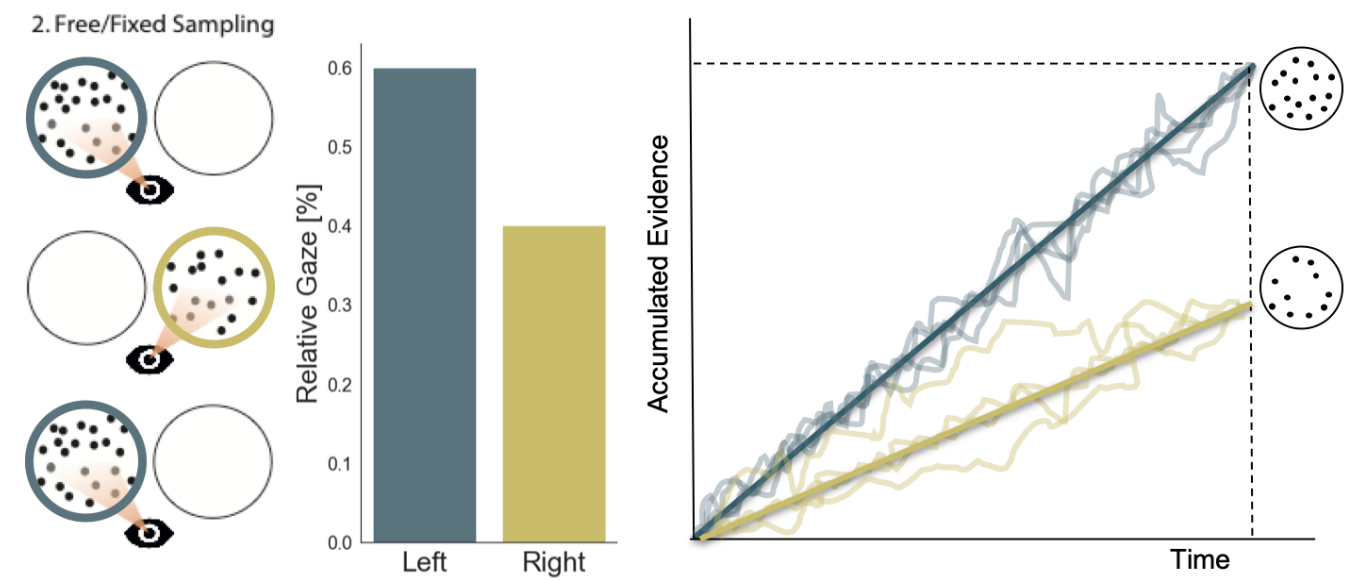

B
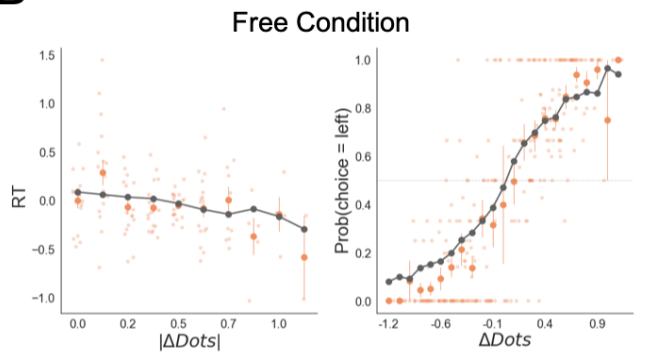

C
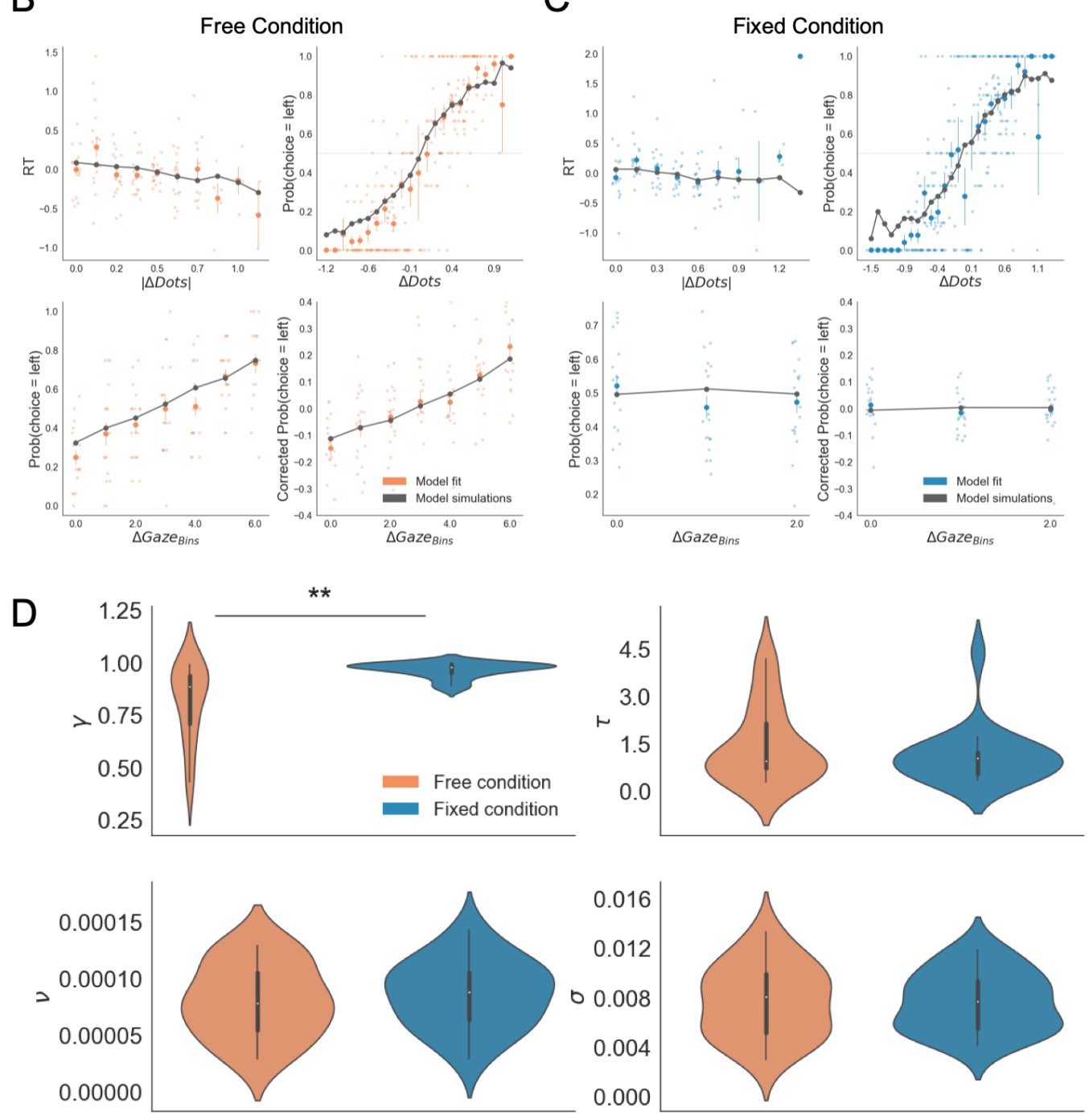

Figure 5. Gaze impacted evidence accumulation (for the $2^{\text {nd }}$ choice) more strongly in the free than in the fixed sampling condition. (A) Free and fixed sampling condition trials were fitted separately using a Gaze-weighted Linear Accumulator Model (GLAM). In this model there are two independent accumulators for each option (left and right) and the decision is made once one of them reaches a threshold. Accumulation rate was modulated by gaze times, when gaze bias parameter is lower than $1(\gamma<1)$. In that 
case, the accumulation rate will be discounted depending on $\gamma$ and the relative gaze time to the items, within the trials. Gaze information from the free sampling trials, and presentation times from the fixed sampling trials were used to fit the models. The panel depicts an example trial: patch sampling during phase 2 (left panel) is used to estimate the relative gaze for that trial (central panel), and the resulting accumulation process (right panel) Notice GLAM ignores fixations dynamics and uses a constant accumulation term within trial (check Methods for further details). The model predicted the behaviour in free (B) and fixed (C) sampling conditions. The four panels present four relevant behavioural relationships comparing model predictions and overall participant behaviour: (top left) response time was faster (shorter RT) when the choice was easier (i.e. bigger differences in the number of dots between the patches); (top right) probability of choosing the left patch increased when the number of dots was higher in the patch at the left side $(\Delta$ Dots

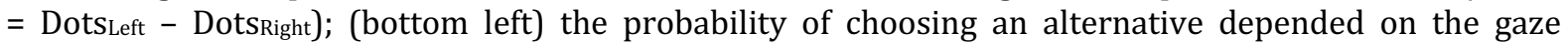
difference $\left(\left(\Delta\right.\right.$ Gaze $\left.=g_{\text {Left }}-g_{\text {Right }}\right)$; and (bottom right $)$ the probability of choosing an item that was fixated longer than the other, corrected by the actual evidence $\Delta$ Dots, depicted a residual effect of gaze on choice. Notice that in the free condition, the model predicted an effect of gaze on choice in a higher degree than in the fixed condition. Solid dots depict the mean of the data across participants in both conditions. Lighter dots present the mean value for each participant across bins. Solid grey lines show the average for model simulations. Data z-scored/binned for visualisation. (D) GLAM parameters fitted at participant level for free and fixed sampling conditions. Free sampling condition presented a higher gaze bias than fixed sampling, while no significant differences were found for the other parameters. $\gamma$ : gaze bias; $\tau$ : evidence scaling; $v$ : drift term; $\sigma$ : noise standard deviation. ${ }^{* *}$ : $\mathrm{p}<0.01$

\section{Discussion}

In this work we have demonstrated that a form of confirmation bias exists in active information sampling, and not just in information weighting as previously shown. Using two novel experiments we showed that this effect is robust for simple perceptual choice. Critically we show that this sampling bias affects future choice. Furthermore, we demonstrated that this effect is only present in the free-sampling condition, showing that agency is essential for biased sampling to have an effect on subsequent choice.

Preference for confirmatory evidence has previously mostly been studied in the context of strongly held political, religious or lifestyle beliefs, and not in perceptual decisionmaking (Bakshy et al., 2015; Hart et al., 2009; Lord et al., 1979; Nickerson, 1998; Stroud, 2008; Wason, 1960,1968). Our results, together with the recent work of others (Talluri, Urai et al., 2018; Palminteri et al., 2017; Lefebvre et al., 2020), show that confirmation bias in information search is present even in decisions where the beliefs formed by participants are not meaningful to their daily lives. This suggests that confirmation bias might be a fundamental property of information sampling, existing irrespective of how important the belief is to the agent.

Recent findings suggest that biases in information sampling might arise from Pavlovian approach, a behavioural strategy that favours approaching choice alternatives associated with reward (Hunt et al., 2016; Rutledge et al., 2015). Furthermore, the number of hypotheses an individual can consider in parallel is likely to be limited (Tweney et al., 1980). As such, it may be advantageous to first attempt to rule out the dominant hypothesis before going on to sample from alternative options. In this vein, the sampling bias we see could be the solution to an exploit-explore dilemma in which the decisionmaker must decide when to stop 'exploiting' a particular hypothesis and instead 'explore' different ones. 
434 A key novel feature of this task is that participants were able to freely sample information

435 between choice phases, providing a direct read-out of confirmation bias in the active 436 sampling decisions made by the participants. Previous accounts of confirmation bias in 437 perceptual choice have instead focused on altered weighting of passively viewed 438 information as a function of previous choice (Bronfman et al., 2015; Rollwage et al., 2020; 439 Talluri, Urai et al., 2018). However, from these findings it remained unclear to what extent 440 this bias manifests in the processing of information compared to active sampling of 441 information. Our findings show that active information sampling plays a key role in the 442 amplification of beliefs from one decision to the next, and that changes in evidence 443 weighting likely only account for part of observed confirmation bias effects.

444

445

We show that confidence modulated the confirmation bias effect, such that choices made with higher confidence, led to increased sampling of the chosen option and an increased likelihood of choosing the same option again in the second choice phase. This shows that the strength with which a belief is held determines the size of the confirmation bias in active information sampling. Confidence has previously been shown to affect the integration of confirmatory evidence as reflected in MEG recordings of brain activity during evidence accumulation (Rollwage et al., 2020). Furthermore, recent work in economics and neuroscience have given theoretical and experimental proof of a relationship between overconfidence, and extreme political beliefs (Ortoleva \& Snowberg, 2015; Rollwage et al., 2018). Our results suggest altered information sampling could be the missing link between these two phenomena. Specifically, given that we have shown that increased confidence leads to increased confirmation bias, it follows that overconfidence in a belief would lead to increased sampling of confirmatory evidence in line with that belief, which in turn would lead to even higher confidence.

459

460

We also show that it is not sufficient that agents freely make a choice for that choice to bias future decisions. We do not see an effect of the first choice on subsequent choices when the intermediate sampling phase is fixed by the experimenter, suggesting the integration of information into beliefs is dependent on whether the agent has actively chosen to sample this information. In other words, a sense of agency appears to impact the extent to which new information influences future choice (Hassall et al., 2019; Chambon et al., 2020), and making a choice may not necessarily lead to confirmation bias if it is not followed by active decisions to sample evidence in line with that choice. Our results are in line with Chambon et al. (2020), in which confirmation bias in learning rates was only present when participants were able to freely choose between stimuli and not when these choices were fixed by the experimenter. In their task, though, choice and information gain were not separated by design, meaning information sampling was only possible from outcome feedback after a choice was made. Our results expand on these findings, by showing that choice commitment also affects subsequent decisions to sample, and that the resulting biased sampling influences future choices. This means that active information sampling is likely to play a central role in the propagation of confirmation bias across decisions, as seen in our descriptive economic model (S6).

Additionally, the results from the GLAM model show that, in line with previous studies (Krajbich et al., 2010; Krajbich \& Rangel, 2010; Sepulveda et al., 2020; Tavares et al., 2017; Thomas et al. 2019), a specific boost in the accumulation of evidence of the visually attended items was found in the free sampling condition. Conversely, a disconnection 
condition (i.e. the absence of gaze bias in GLAM implies that visual fixations did not affect evidence integration when the sampling was not controlled by the decision-maker). One explanation for this result is that attentional allocation itself is directed towards the options that the participants reckon more relevant for the task to be performed (Sepulveda et al., 2020). In our experiment the goal of the task was theoretically identical for the first and second choice (i.e. to find the patch with more dots). However, in agreement with the ideas of our descriptive economic model, it could be that participants perceived the goal of the second choice to be slightly different from the goal of the first: in the second case they had to verify whether their initial choice was correct, as well as finding the patch with the most dots. This immediately bestowed the chosen option with higher relevance and then more attention (consequently boosting evidence accumulation). On the other hand, since in the fixed sampling condition participant's attention allocation is not necessarily associated with their goals, the difference in display time of the items is ignored or cannot be consistently integrated in their decision process. An alternative hypothesis is that gaze allocation itself is used as additional evidence in favour of a choice alternative in a way similar to previous choice as in our descriptive model (S6). This would mean that when an agent has previously attended to a choice alternative, this is used as evidence in favour of that option in and of itself. Therefore, when gaze allocation is not under the agent's control, as in the fixed-viewing condition, it is not used to inform choice.

Our findings imply that agency plays a clear role in evidence accumulation, and consequently in confirmation bias. It also suggests that common experimental designs in which information is provided by the experimenter and passively sampled by the participant might not be an ecological way to study decision-making. These tasks mask the potentially large effect of active information search on belief formation and choice. Pennycook et al. (2021) recently showed that people were less likely to share false information online if they had been asked to rate the accuracy of a headline just previously. It may therefore be possible to reduce confirmation bias in information search in a similar way by priming participants to attend more to accuracy instead of confirmatory evidence. More complex behavioural tasks are required to improve our understanding of the different drivers of information sampling and how sampling in turn guides future choice (Gottlieb \& Oudeyer, 2018).

To summarise our findings, we observed that participants sampled more information from chosen options in a perceptual choice paradigm and that this sampling bias predicted subsequent choice. Asymmetric sampling in favour of the chosen alternative was stronger the higher participants' confidence in this choice. Furthermore, the effect of information on subsequent choice was only seen in a version of the task where participants could sample freely, suggesting agency plays an important role in the propagation of strongly held beliefs over time. These findings suggest that confirmatory information processing might stem from a general information sampling strategy used to seek information to strengthen prior beliefs rather than from altered weighting during evidence accumulation only, and that active sampling is essential to this effect. Biased sampling may cause a continuous cycle of belief reinforcement that can be hard to break. Improving our understanding of this phenomenon can help us better explain the roots of extreme political, religious and scientific beliefs in our society. 


\section{Participants}

Experiment 1. 30 participants took part in this study. We excluded two participants from the analysis, because they gave the highest possible confidence rating on more than $75 \%$ of trials. Participants received a $£ 10$ show-up fee in addition to a monetary reward between $£ 0$ and $£ 6$, which could be gained in the task. Participants had normal or corrected-to-normal vision, and no psychiatric or neurological disorders. We obtained written informed consent from all participants before the study. This experiment was approved by the University of Cambridge Psychology Research Ethics Committee.

Experiment 2. 23 participants completed this experiment, of which two were excluded from the analysis, because they gave the highest possible confidence rating on more than $75 \%$ of trials. Another three participants were excluded because their confidence was a poor predictor of their accuracy in the task (lower than two standard deviations under the mean coefficient predicting accuracy in a logistic regression). Participants were reimbursed $£ 30$ for their time as well as an additional amount between $£ 0$ and $£ 20$ that could be gained in the task. Participants had normal or corrected-to-normal vision, and no psychiatric or neurological disorders. We obtained written informed consent from all participants before the study. This experiment was approved by the University of Cambridge Psychology Research Ethics Committee.

\section{Behavioural Task}

Experiment 1. The computer task used in this study consisted of 200 trials. Participants were asked to make binary choices between dot patches using the arrow keys on the keyboard. They then reported their confidence in this decision on a continuous rating scale. In the subsequent sampling phase, participants were given the opportunity to look at the patches again for $4000 \mathrm{~ms}$. By using the left and right arrow keys they could alternate as often as they wanted between viewing either dot patch within the given time. Afterwards, participants were prompted to make a second choice for the same pair of patches and give another confidence judgment. Participants were constantly reminded of their initial choice by a green tick next to the chosen patch. Participants received no feedback about the correctness of their choice. None of the choices made or confidence ratings given were time-constrained. One of the patches always contained 50 dots, and the other a variable amount of dots. We calibrated the dot difference between the two patches such that accuracy level within each participant was maintained at $70 \%$ throughout the task using a one-up two-down staircase procedure (Fleming \& Lau, 2014) The task was programmed using Python 2.7.10 and PsychoPy (Peirce, 2007).

Experiment 2. A similar task was used in the second experiment, except that in this task participants' responses were elicited through eye movements. To make a choice they looked at one of the patches and to rate their confidence they looked at a position inside a rating scale. The sampling phase between the two choices randomly varied in this task, and was either 3000,5000 , or $7000 \mathrm{~ms}$. This was not cued to the participants, so at the start of a sampling phase they did not know how much time they would be given to sample the patches. Sampling was gaze-contingent such that the dots were only visible when the participant fixated inside a patch and participants could only see one patch at a time. Furthermore, we introduced a control condition in which participants were not free to sample the circles however they liked during the sampling phase. Instead, in one-third 
580

581

582

583

584

585

586

587

588

589

590

591

592

593

594

595

596

597

598

599

600

601

602

603

604

605

606

607

608

609

610

611

612

613

614

615

616

617

618

619

620

621

622

623

624

625

626

627

628

of trials the patches were shown for an equal amount of time each and in two-thirds of trials one patch was shown three times longer than the other $(50 \%$ of trials the left was shown longer, $50 \%$ of trials the right was shown longer). Participants were constantly reminded of their initial choice by the circle surrounding the chosen patch changing color. The first experiment conducted using this task was a behavioural experiment in which participants recorded their responses using the arrow keys on a computer. In the second experiment, participants responded using eye movements. In the second experient, participants took part in two sessions, each consisting of 189 trials. In one session they performed the main task and in the other the control condition of the task. The order of these two sessions was pseudo-random. This experiment was programmed using the SR Research Experiment Builder version 1.10.1630 (SR Research Experiment Builder, 2017).

\section{Eye-tracking}

In experiment 2, we recorded eye movements at a rate of 1,000Hz using an EyeLink 1000 Plus eye-tracker. Areas of Interest (AI) for the eye tracking analyses were pre-defined as two squares centered on the gaze-contingent circles in the experiment. The sides of the squares were the same as the diameter of the gaze-contingent circles. For each decision period we derived the total dwell time in each AI from the eye-tracking data. The computer used in this experiment had a screen size of $68.58 \times 59.77 \mathrm{~cm}$ and participants were seated $60 \mathrm{~cm}$ away from the screen.

\section{Analyses}

We studied the effect of choice on the time spent on each of the two stimuli using paired sample t-tests on the mean sampling times spent on each stimulus from each participant. Trials with the shortest sampling phase length of $3000 \mathrm{~ms}$ in experiment 2 were excluded from all analyses, because it became apparent that this time was too short for participants to be able to saccade to each circle more than once.

Hierarchical models. Hierarchical regression models were conducted using the lme4 package in R (Bates et al., 2015; Gelman \& Hill, 2006). We computed degrees of freedom and p-values with the Kenward-Roger approximation, using the package pbkrtest (Halekoh \& Hojsgaard, 2014). We predicted the sampling time difference between the two circles using a hierarchical linear regression model. To predict choice in the second choice phase, hierarchical logistic regressions were used predicting the log odds ratio of picking the left circle on a given trial. Confidence and sampling time were z-scored on the participant level. For detailed results and model comparisons, see supplemental materials S1-S2, S7.

Attentional model - GLAM. The Gaze-weighted Linear Accumulator Model (Thomas et al., 2019; Molter et al., 2019) is part of the family of linear stochastic race models in which different alternatives ( $\mathrm{i}$; left or right) accumulate evidence (Ei) until a decision threshold is reached by one of them, determining the chosen alternative. The accumulator for an individual option was defined by the expression:

$$
E_{i}(t)=E_{i}(t-1)+v R_{i}+\epsilon_{t} \text { with } \epsilon_{t} \sim N(0, \sigma) \text { and } E_{i}(t=0)=0
$$

A drift term $(v)$ controlled the speed of relative evidence $\left(R_{i}\right)$ integration and noise was integrated with normal distribution (zero-centered and with standard deviation, $\sigma$ ). $\mathrm{R}_{\mathrm{i}}$ expressed the amount of evidence that was accumulated for item $i$ at each time point $t$. 
629

630

631

632

633

634

635

636

637

638

639

640

641

642

643

644

645

646

647

648

649

650

651

652

653

654

655

656

657

658

659

660

661

662

663

664

665

666

667

668

669

670

671

672

673
This was calculated as follows. We denote by $g_{i}$, the relative gaze term, calculated as the proportion of time that participants observed item $\mathrm{i}$ :

$g_{i}=\frac{D T_{i}}{D T_{1}+D T_{2}}$

with DT as the dwelling time for item i during an individual trial. Let $r_{i}$ denote the evidence (in this study evidence corresponded to number of dots presented for each option) for item $\mathrm{i}$. We can then define the average absolute evidence for each item $\left(\mathrm{A}_{\mathrm{i}}\right)$ during a trial:

$$
A_{i}=g_{i} r_{i}+\left(1-g_{i}\right) \gamma r_{i}
$$

This formulation considers a multiplicative effect of the attentional component over the evidence, capturing different rates of integration when the participant was observing item i or not (unbiased and biased states, respectively). The parameter $\gamma$ was the gaze bias parameter: it controlled the weight that the attentional component had in determining absolute evidence. When $\gamma=1$, accumulation was the same in biased and unbiased states, i.e. gaze did not affect the accumulation of evidence. When $\gamma<1, A_{i}$ was discounted for the biased condition, resulting in higher probability of choosing items that had been gazed at longer. When $\gamma<0$, the model assumed a leak of evidence when the item was not fixated. Therefore, the relative evidence of item $\mathrm{i}, \mathrm{R}_{\mathrm{i}}^{*}$, was given by:

$R_{i}^{*}=A_{i}-\max _{j \neq i} A_{j}=A_{i}-A_{j} \rightarrow R_{\text {right }}^{*}=-R_{\text {left }}^{*}$

Since our experiment considers a binary choice, while the original formulation of the model (Thomas et al., 2019) proposed for more than two alternatives, $\mathrm{R}_{\mathrm{i}}{ }^{*}$ was reduced to subtract the average absolute evidence of the other item. Therefore, for the binary case, $\mathrm{R}_{\mathrm{i}}^{*}$ for one item was the additive inverse of the other. For example, if the left item had the lower evidence, we would have $\mathrm{R}_{\text {left }}^{*}<0$ and $\mathrm{R}_{\text {right }}^{*}>0$. The difference in signals captured by $\mathrm{R}_{\mathrm{i}}^{*}$ was scaled by a logistic transformation. The model assumed an adaptive representation of the relative decision signals, which is maximally sensitive to marginal differences in absolute decision signals:

$$
R_{i}=\frac{1}{1+\exp \left(-\tau R_{i}^{*}\right)}
$$

The temperature parameter $\tau$ of the logistic function controlled the sensitivity of the transformation. Larger values of $\tau$ indicate stronger sensitivity to small differences in absolute evidence $\left(A_{i}\right)$. Given that $R_{i}$ represents an average of the relative evidence across the entire trial, the drift rate in $E_{i}$ can be assumed to be constant, which enables the use of an analytical solution for the passage of time density (for details see Thomas et al., 2019, Molter et al., 2019). Notice that unlike the aDDM (Krajbich et al., 2010), GLAM does not deal with the dynamics of attentional allocation process in choice. In summary, the GLAM model considers four free parameters: $v$ (drift term), $\gamma$ (gaze bias), $\tau$ (evidence scaling), and $\sigma$ (normally distributed noise standard deviation). 
674 The model fit with GLAM was implemented at a participant level in a Bayesian framework

675 using PyMC3 (Salvatier et al., 2016). Uniform priors were used for all the parameters:

$676 \quad v \sim$ Uniform $\left(1^{-6}, 0.01\right)$

$677 \gamma \sim$ Uniform $(-1,1)$

$678 \sigma \sim$ Uniform $\left(1^{-6,5)}\right.$

$679 \tau \sim$ Uniform $(0,5)$

680

681

682

We fitted the model for each individual participant and for free and fixed sampling conditions in experiment 2 separately. To model participants' behaviour, we used as

683 input for GLAM the reaction times (RT) and choices obtained from phase 3, and relative

684

685 gaze for left and right alternatives for each trial during sampling phase 2. For fixed sampling trials the presentation times of the dot patches were used to calculate the relative gaze time. For both conditions, model fit was performed only on even-numbered trials using Markov-Chain-Monte-Carlo sampling, we used implementation for No-UTurn-Sampler (NUTS), four chains were sampled, 1000 tuning samples were used, and 2000 posterior samples were used to estimate the model parameters. The convergence was diagnosed using the Gelman-Rubin statistic $(|\hat{R}-1|<0.05)$ for the four parameters $(v, \gamma, \sigma$, and $\tau$ ). Considering all the individual models (18 participants), we found divergences in $\sim 16 \%$ of the estimated parameters. We removed the participants that presented divergent parameters (4 participants) to check whether the results we found were driven by these data. The significantly higher gaze bias in free-viewing condition was maintained even after removing these participants (see supplemental materials, S5.4). Model comparison was performed using Watanabe-Akaike Information Criterion

\section{Acknowledgements}

710 These studies were funded by the Wellcome Trust and Royal Society (Henry Dale 711 Fellowship no. 102612/Z/13/Z to B.D.M.). P.S. was funded by the Chilean National 712 Agency for Research and Development (ANID)/Scholarship Program/DOCTORADO BECAS CHILE/2017 - 72180193. The authors declare no conflict of interest. 
Bakshy, E., Messing, S., \& Adamic, L. A. (2015). Exposure to ideologically diverse news and opinion on Facebook. Science, 348(6239), 1130-1132. https://doi.org/10.1126/science.aaa1160

Bates, D., KliegI, R., Vasishth, S., \& Baayen, H. (2015). Parsimonious Mixed Models. ArXiv. http://arxiv.org/abs/1506.04967

Bennett, W. L., \& Iyengar, S. (2008). A New Era of Minimal Effects? The Changing Foundations of Political Communication. Journal of Communication, 58(4), 707-731. https://doi.org/10.1111/j.1460-2466.2008.00410.x

Bogacz, R., Wagenmakers, E.-J., Forstmann, B. U., \& Nieuwenhuis, S. (2010). The neural basis of the speed-accuracy tradeoff. Trends in Neurosciences, 33(1), 10-16. https://doi.org/10.1016/j.tins.2009.09.002

Boldt, A., Blundell, C., \& De Martino, B. (2019). Confidence modulates exploration and exploitation in value-based learning. Neuroscience of Consciousness, 2019(1), niz004. https://doi.org/10.1093/nc/niz004

Bronfman, Z. Z., Brezis, N., Moran, R., Tsetsos, K., Donner, T., \& Usher, M. (2015). Decisions reduce sensitivity to subsequent information. Proceedings of the Royal Society $B$ : Biological Sciences, 282(1810), 20150228. https://doi.org/10.1098/rspb.2015.0228

734 Carrasco, M., Ling, S., \& Read, S. (2004). Attention alters appearance. Nature Neuroscience, 7(3), 308-313. https://doi.org/10.1038/nn1194

736 Chambon, V., Théro, H., Vidal, M., Vandendriessche, H., Haggard, P., \& Palminteri, S. (2020). determined versus imposed choices. Nature Human Behaviour, 4(10), 1067-1079. 
740 Desender, K., Murphy, P., Boldt, A., Verguts, T., \& Yeung, N. (2019). A Postdecisional Neural Marker of Confidence Predicts Information-Seeking in Decision-Making. The Journal of Neuroscience, 39(17), 3309-3319. https://doi.org/10.1523/JNEUROSCI.262018.2019

Eliaz, K., \& Schotter, A. (2007). Experimental Testing of Intrinsic Preferences for NonInstrumental Information. American Economic Review, 97(2), 166-169. https://doi.org/10.1257/aer.97.2.166

Fleming, S. M., \& Lau, H. C. (2014). How to measure metacognition. Frontiers in Human Neuroscience, 8. https://doi.org/10.3389/fnhum.2014.00443

Folke, T., Jacobsen, C., Fleming, S. M., \& Martino, B. D. (2017). 1 Explicit representation of confidence informs future 2 value-based decisions. Nature Human Behaviour, 1(0002).

Gelman, A., \& Hill, J. (2006). Data Analysis Using Regression and Multilevel/Hierarchical Models. Cambridge University Press.

Gesiarz, F., Cahill, D., \& Sharot, T. (2019). Evidence accumulation is biased by motivation: A computational account. PLOS Computational Biology, 15(6), e1007089. https://doi.org/10.1371/journal.pcbi.1007089

Gottlieb, J., \& Oudeyer, P.-Y. (2018). Towards a neuroscience of active sampling and curiosity. Nature Reviews Neuroscience, 19(12), 758-770. https://doi.org/10.1038/s41583-018-0078-0 Journal of Statistical Software, 59(9), 1-30. 
763

764

765

766

767

768

769

770

771

772

773

774

775

776

777

778

779

780

781

782

783

784

785

Hart, W., Albarracín, D., Eagly, A. H., Brechan, I., Lindberg, M. J., \& Merrill, L. (2009). Feeling validated versus being correct: A meta-analysis of selective exposure to information. Psychological Bulletin, 135(4), 555-588. https://doi.org/10.1037/a0015701

Hassall, C. D., Hajcak, G., \& Krigolson, O. E. (2019). The importance of agency in human reward processing. Cognitive, Affective, \& Behavioral Neuroscience, 19(6), 14581466. https://doi.org/10.3758/s13415-019-00730-2

Hunt, L. T., Malalasekera, W. M. N., de Berker, A. O., Miranda, B., Farmer, S. F., Behrens, T. E. J., \& Kennerley, S. W. (2018). Triple dissociation of attention and decision computations across prefrontal cortex. Nature Neuroscience, $21,1471-1481$.

Hunt, L. T., Rutledge, R. B., Malalasekera, W. M. N., Kennerley, S. W., \& Dolan, R. J. (2016). Approach-Induced Biases in Human Information Sampling. PLOS Biology, 14(11), e2000638. https://doi.org/10.1371/journal.pbio.2000638

Kobayashi, K., \& Hsu, M. (2019). Common neural code for reward and information value. Proceedings of the National Academy of Sciences, 116(26), 13061-13066. https://doi.org/10.1073/pnas.1820145116

Kobayashi, K., Ravaioli, S., Baranès, A., Woodford, M., \& Gottlieb, J. (2019). Diverse motives for human curiosity. Nature Human Behaviour, 3(6), 587-595. https://doi.org/10.1038/s41562-019-0589-3

Krajbich, I., \& Rangel, A. (2011). Multialternative drift-diffusion model predicts the relationship between visual fixations and choice in value-based decisions. Proceedings of the National Academy of Sciences, 108(33), 13852-13857.

Krajbich, I., Armel, C., \& Rangel, A. (2010). Visual fixations and the computation and comparison of value in simple choice. Nature Neuroscience, 13(10), 1292-1298. 
786 Lefebvre, G., Summerfield, C., \& Bogacz, R. (2020). A normative account of confirmatory biases during reinforcement learning. bioRxiv. doi: https://doi.org/10.1101/2020.05.12.090134

Lord, C. G., Ross, L., \& Lepper, M. R. (1979). Biased Assimilation and Attitude Polarization: The Effects of Prior Theories on Subsequently Considered Evidence. Journal of Personality and Social Psychology, 37(11), 2098-2109.

Meyer, R. J., \& Shi, Y. (1995). Sequential Choice Under Ambiguity: Intuitive Solutions to the Armed-Bandit Problem. Management Science, 41(5), 817-834.

Molter, F., Thomas, A. W., Heekeren, H. R., \& Mohr, P. N. (2019). GLAMbox: A Python behaviour. PloS One, 14(12), e0226428.

Nickerson, R. S. (1998). Confirmation Bias: A Ubiquitous Phenomenon in Many Guises. Review of General Psychology, 2(2), 175-220. Economic Review, 105(2), 504-535. human reinforcement learning: Evidence from counterfactual feedback processing. PLOS Computational Biology, 13(8), e1005684.

Palminteri, S., Wyart, V., \& Koechlin, E. (2017). The importance of falsification in computational cognitive modeling. Trends in cognitive sciences, 21(6), 425-433. 
Peirce, J. W. (2007). PsychoPy-Psychophysics software in Python. Journal of Neuroscience Methods, 6.

811 Pennycook, G., Epstein, Z., Mosleh, M., Arechar, A.A., Eckles, D., \& Rand, D.G. (2021). Shifting attention to accuracy can reduce misinformation online. Nature. https://doi.org/10.1038/s41586-021-03344-2

Pouget, A., Drugowitsch, J., \& Kepecs, A. (2016). Confidence and certainty: Distinct probabilistic quantities for different goals. Nature Neuroscience, 19(3), 366-374.

Rabbitt, P. M. (1966). Errors and error correction in choice-response tasks. Journal of Experimental Psychology, 71(2), 264-272.

Rodriguez Cabrero, J. A. M., Zhu, J.-Q., \& Ludvig, E. A. (2019). Costly curiosity: People pay a price to resolve an uncertain gamble early. Behavioural Processes, 160, 20-25. https://doi.org/10.1016/j.beproc.2018.12.015

Rollwage, M., Dolan, R. J., \& Fleming, S. M. (2018). Metacognitive Failure as a Feature of Those Holding Radical Beliefs. Current Biology, 28(24), 4014-4021.e8. https://doi.org/10.1016/j.cub.2018.10.053 Confidence drives a neural confirmation bias. Nature Communications, 11(1), 2634. https://doi.org/10.1038/s41467-020-16278-6

Rutledge, R. B., Skandali, N., Dayan, P., \& Dolan, R. J. (2015). Dopaminergic Modulation of Decision Making and Subjective Well-Being. Journal of Neuroscience, 35(27), 98119822. https://doi.org/10.1523/JNEUROSCI.0702-15.2015

831 Salem-Garica, N., Palminteri, S., \& Lebreton, M. (2021). The computational origins of 
833 Salvatier, J., Wiecki, T. V., \& Fonnesbeck, C. (2016). Probabilistic programming in Python using PyMC3. PeerJ Computer Science, 2, e55.

835 Schulz, E., \& Gershman, S. J. (2019). The algorithmic architecture of exploration in the human brain. Current Opinion in Neurobiology, 55, 7-14. https://doi.org/10.1016/j.conb.2018.11.003 K. J. (2019). Computational mechanisms of curiosity and goal-directed exploration. ELife, 8, e41703. https://doi.org/10.7554/eLife.41703

841 Sepulveda, P., Usher, M., Davies, N., Benson, A., Ortoleva, P., \& De Martino, B. (2020). Visual attention modulates the integration of goal-relevant evidence and not value. ELife, 9, e60705. https://doi.org/10.1101/2020.04.14.031971

Sharot, T. (2011). The optimism bias. Current Biology, 21(23), R941-R945. https://doi.org/10.1016/j.cub.2011.10.030

SR Research Experiment Builder (1.10.1630). (2017). [Computer software]. SR Research Ltd. decision-making on bandit problems. Journal of Mathematical Psychology, 53(3), 168-179. https://doi.org/10.1016/j.jmp.2008.11.002

850 Stroud, N. J. (2008). Media Use and Political Predispositions: Revisiting the Concept of Selective Exposure. Political Behavior, 30(3), 341-366. https://doi.org/10.1007/s11109-007-9050-9

853 Talluri, B. C., Urai, A. E., Tsetsos, K., Usher, M., \& Donner, T. H. (2018). Confirmation Bias 28(19), 3128-3135.e8. https://doi.org/10.1016/i.cub.2018.07.052 
856 Tarantola, T., Folke, T., Boldt, A., Pérez, O.D., \& De Martino, B. (2021). Confirmation bias optimizes reward learning. bioRxiv. doi: https://doi.org/10.1101/2021.02.27.433214

858 Tavares, G., Perona, P., \& Rangel, A. (2017). The attentional drift diffusion model of simple perceptual decision-making. Frontiers in neuroscience, 11, 468.

860 Thomas, A. W., Molter, F., Krajbich, I., Heekeren, H. R., \& Mohr, P. N. (2019). Gaze bias differences capture individual choice behaviour. Nature Human Behaviour, 3(6), 625635.

863 Tolcott, M. A., Marvin, F. F., \& Lehner, P. E. (1989). Expert decision-making in evolving situations. IEEE Transactions on Systems, Man, and Cybernetics, 19(3), 606-615.

866 Tweney, R. D., Doherty, M. E., Worner, W. J., Pliske, D. B., Mynatt, C. R., Gross, K. A., \& Arkkelin, D. L. (1980). Strategies of Rule Discovery in an Inference Task. Quarterly Journal of Experimental Psychology, 32(1), 109-123. https://doi.org/10.1080/00335558008248237 (2018). Induction and Relief of Curiosity Elicit Parietal and Frontal Activity. The Journal of Neuroscience, 38(10), 2579-2588. https://doi.org/10.1523/JNEUROSCI.2816-17.2018

874 Vandormael, H., Castañón, S. H., Balaguer, J., Li, V., \& Summerfield, C. (2017). Robust sampling of decision information during perceptual choice. Proceedings of the National Academy of Sciences, 114(10), 2771-2776.

877 Wang, M. Z., \& Hayden, B. Y. (2019). Monkeys are Curious about Counterfactual Outcomes. BioRxiv. 
879 Wason, P. C. (1960). On the failure to eliminate hypotheses in a conceptual task. Quarterly Journal of Experimental Psychology, 12(3), 129-140.

881 https://doi.org/10.1080/17470216008416717

Wason, P. C. (1968). Reasoning about a Rule. Quarterly Journal of Experimental Psychology, $20,273-281$. directed and random exploration to solve the explore-exploit dilemma. Journal of Experimental Psychology: General, 143(6), 2074-2081. https://doi.org/10.1037/a0038199

888 Yang, S. C.-H., Wolpert, D. M., \& Lengyel, M. (2016). Theoretical perspectives on active sensing. Current Opinion in Behavioral Sciences, 11, 100-108. https://doi.org/10.1016/j.cobeha.2016.06.009

891 Yeung, N., \& Summerfield, C. (2012). Metacognition in human decision-making: Confidence and error monitoring. Philosophical Transactions of the Royal Society B: Biological 
895

896

897

898

899

900

901

902

903

904

905

906

907

908

909

910

911

912

913

914

915

916

917

918

919

920

921

922

Supplemental Material

$\underline{\text { S1: Hierarchical regression predicting sampling time difference }}$

Hierarchical Regression Model Predicting Sampling Time Difference, Experiment 1

\begin{tabular}{lllll} 
Predictor & Coefficient & SE & t-value & p-value \\
\hline Intercept & -0.27 & 0.03 & -8.48 & $<0.0001$ \\
Choice & 0.51 & 0.05 & 9.64 & $<0.0001$ \\
Dot Difference & 0.05 & 0.004 & 13.02 & $<0.0001$ \\
Confidence & -0.08 & 0.03 & -2.88 & 0.007 \\
Choice $x$ & 0.19 & 0.04 & 5.26 & $<0.0001$ \\
Confidence & & & &
\end{tabular}

This model was run using data from experiment 1 . Confidence was z-scored per participant. It shows that choice is a significant predictor of sampling time difference between the chosen and unchosen circles, such that participants were likely to view the circle they chose for a longer time in the sampling phase. Furthermore, the interaction between choice and confidence was a significant predictor, signifying that the more confident participants were in their choice, the more biased their sampling was in favour of that choice. Dot difference also significantly predicts sampling time: participants sampled the circle containing the most dots for longer. Note that this variable shares some variance with the choice variable.

\section{Hierarchical Regression Model Predicting Sampling Time Difference, Experiment 2}

$\begin{array}{lllll}\text { Predictor } & \text { Coefficient } & \text { SE } & \text { t-value } & \text { p-value } \\ \text { Intercept } & -0.15 & 0.06 & -2.63 & 0.017 \\ \text { Choice } & 0.30 & 0.10 & 2.90 & 0.01 \\ \text { Dot Difference } & 0.04 & 0.005 & 9.21 & <0.0001 \\ \text { Confidence } & -0.09 & 0.04 & -2.43 & 0.03 \\ \text { Choice } x & 0.21 & 0.05 & 4.29 & 0.0005 \\ \text { Confidence } & & & & \end{array}$

We also ran this model using data from experiment 2, excluding control condition trials in which sampling time difference was fixed. Confidence was z-scored per participant. Again, choice, the interaction term between choice and confidence and dot difference were all significant predictors of the difference in sampling time between the two circles. 


\section{S2: Hierarchical logistic regression predicting change of mind}

\section{Hierarchical Logistic Regression Model Predicting Change of Mind, Experiment 1}

\begin{tabular}{lllll} 
Predictor & Coefficient & SE & z-value & p-value \\
\hline Intercept & -1.18 & 0.21 & -5.65 & $<0.0001$ \\
Sampling Bias & -1.24 & 0.08 & -11.0 & $<0.0001$ \\
Dot Difference & -0.24 & 0.11 & -3.06 & 0.002 \\
Confidence & -0.58 & 0.06 & -10.12 & $<0.0001$
\end{tabular}

This model was run using data from experiment 1. Using a hierarchical logistic regression we predicted the log odds ratio that the participant changed their mind in the second decision phase on a given trial. Sampling bias and confidence were z-scored per participant. Convergence issues were addressed by square-root-transforming dot difference. Sampling bias is defined as the difference between the amount of time the initially chosen vs unchosen circles were sampled. This variable negatively predicts change of mind, meaning the more the chosen circle was sampled, the less likely participants were to change their mind. Absolute dot difference, which is a measure of trial difficulty, negatively predicts change of mind, such that participants were less likely to change their mind when the trial was easier. Finally, confidence was a negative predictor of change of mind; the higher confidence in the initial choice, the less likely participants were to change their mind.

\section{Hierarchical Logistic Regression Model Predicting Change of Mind, Experiment 2}

\begin{tabular}{lllll} 
Predictor & Coefficient & SE & z-value & p-value \\
\hline Intercept & -1.01 & 0.28 & -3.62 & 0.0002 \\
Sampling Bias & -1.40 & 0.20 & -7.07 & $<0.0001$ \\
Dot Difference & -0.26 & 0.09 & -2.72 & 0.007 \\
Confidence & -0.57 & 0.06 & -8.86 & $<0.0001$ \\
Fixed Sampling & -0.10 & 0.14 & -0.67 & 0.50 \\
Sampling Bias & 1.44 & 0.20 & 7.24 & $<0.0001$ \\
x Fixed & & & & \\
Sampling & & & &
\end{tabular}

A similar model to that presented was run on the data from experiment 2 . Because this experiment included a control condition in which sampling was fixed, we included a dummy variable in this model coding whether the trial was in the control condition or not. Sampling bias, dot difference, and confidence were all significant negative predictors of change of mind. However, an interaction term between 'Fixed Sampling' (the control condition) and sampling bias was significantly positive, and approximately the same size as the main effect of sampling bias on change of mind. This means that in control condition trials, the effect of sampling bias on change of mind disappears. 
In experiment 2, the length of the sampling phase (phase II) was varied to study the emergence of a sampling bias over time. The sampling phase randomly varied and was either 3000,5000 or $7000 \mathrm{~ms}$. In the experiment, participants were forced to look at each circle at least once during the sampling phase, otherwise the trial was invalidated. We decided to exclude trials with the shortest sampling phase length of $3000 \mathrm{~ms}$, because it became apparent that participants were not able to allocate this time beyond looking at each patch once.

967
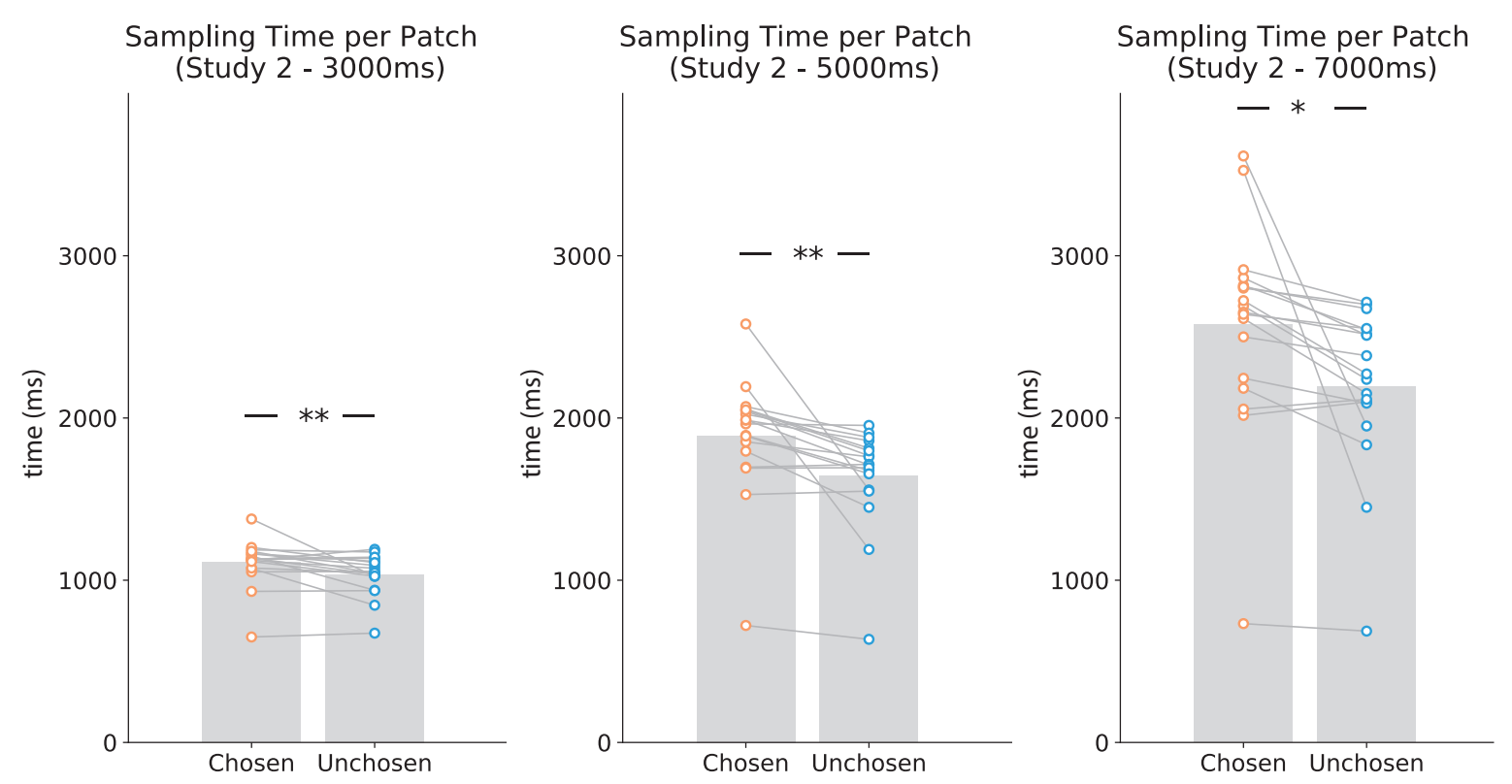

Figure S3.1: Mean sampling time spent viewing the initially chosen and unchosen patch in the sampling 971 phase in studies 2 for each sampling phase length. Data points represent individual participants. 


\section{Sampling bias only affects choice in free sampling trials}

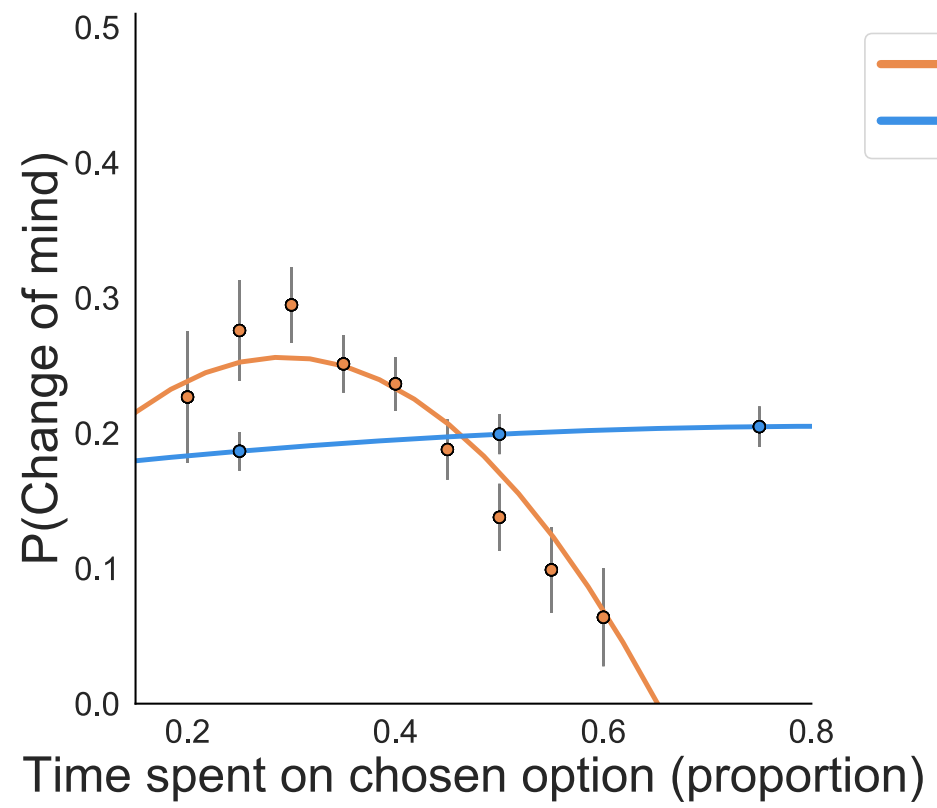

Figure S4.1 In Figure 4D, we plotted the probability of changes of mind as a function of actual gaze time by participants in the fixed viewing condition. Even though stimulus presentation was fixed, participants could still choose to saccade back to the central fixation cross before the end of stimulus presentation. Therefore, actual gaze time is a better reflection of information processing in this condition. Here, we are plotting stimulus presentation time in the fixed sampling condition instead. The same lack of effect of sampling bias on changes of mind can be seen here in the fixed sampling condition as in Figure 4. 
bioRxiv preprint doi: https://doi.org/10.1101/2021.06.29.450332; this version posted June 30, 2021. The copyright holder for this preprint (which was not certified by peer review) is the author/funder, who has granted bioRxiv a license to display the preprint in perpetuity. It is made available under aCC-BY-NC-ND 4.0 International license.

A

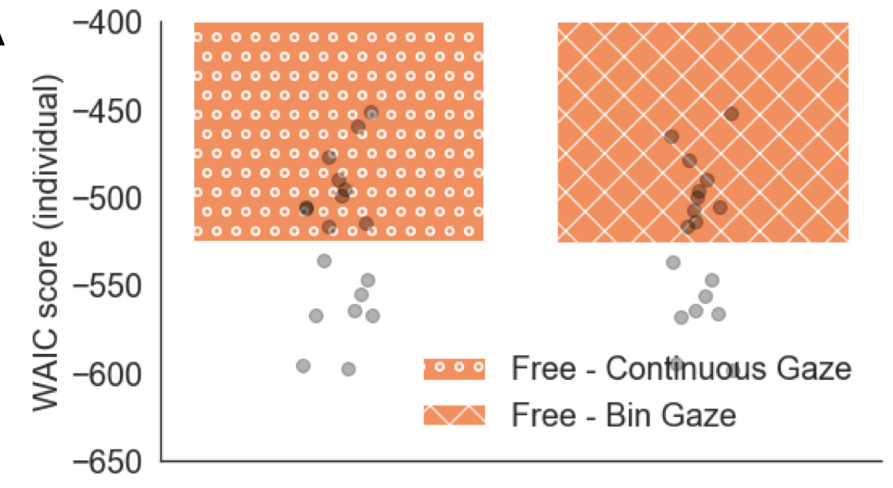

B
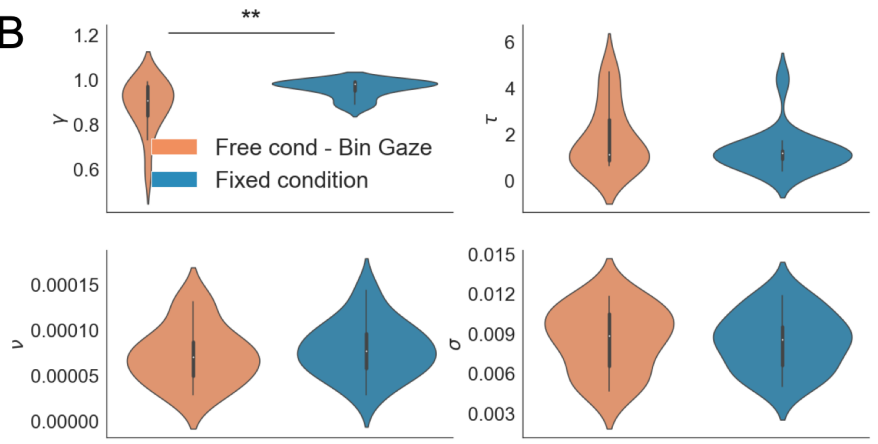

C
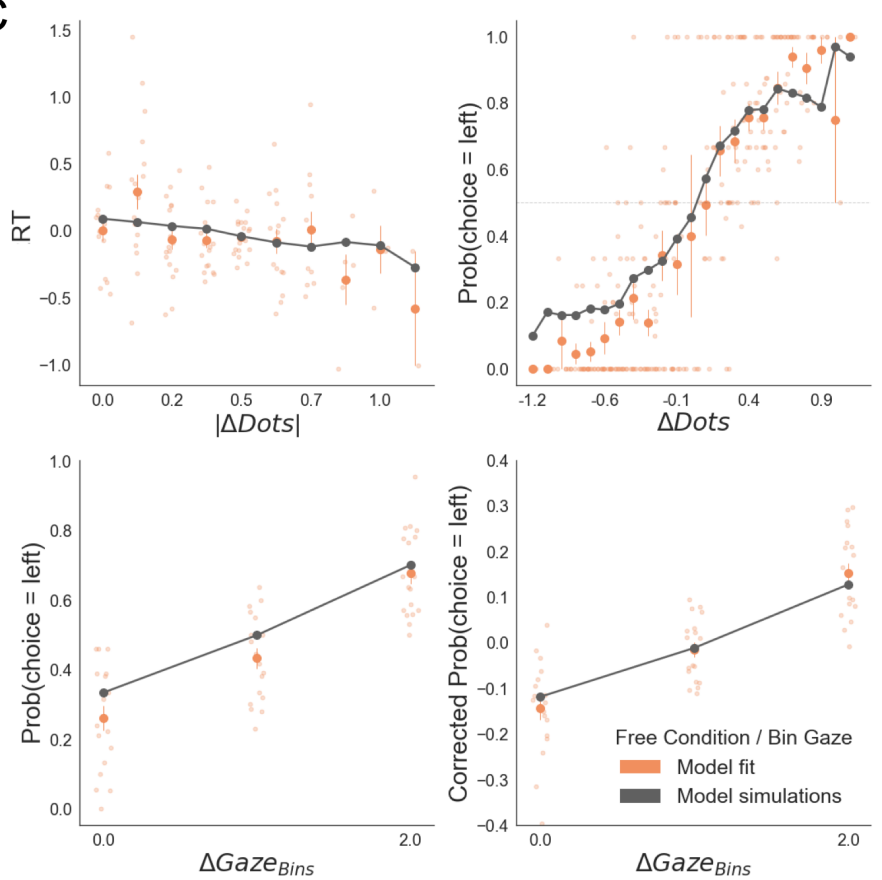

Figure S5.1. GLAM results with down-sampled gaze information in the free sampling condition. To check whether the results reported in the main text are an artefact of the low variability in relative gaze in fixed sampling trials, we reduced the variability of free sampling trials to only 3 bins. This replicated the number of bins in the gaze allocation presented in our fixed-viewing trials. GLAM was fitted using these data. (A) Model comparison between the GLAM models in the free sampling condition fitted using original gaze data (continuous gaze) and the down sampled gaze information (bin gaze). We found no significant difference between the model fit scores in both cases (Mean $\mathrm{WAIC}_{\text {Free/Continuous }}=-524.58$; Mean $\mathrm{WAIC}_{\text {Free/Bin }}=$ $\left.-525.12 ; \mathrm{t}_{17}=1.46, \mathrm{p}=0.16, \mathrm{~ns}\right)$. Model fitting was performed at a participant level. WAIC scores are 
parameters resulting from Bin Gaze model fit. We replicated the findings in the main text, with a higher gaze bias (lower $\gamma$ parameter) in the free sampling condition (Mean $\gamma_{\text {Free }}=0.81$, Mean $\gamma_{\text {Fixed }}=0.96, t_{17}=-$ 3.268; $\mathrm{p}<0.01$ ). No significant differences were found for the other parameters. $\gamma$ : gaze bias; $\tau$ : evidence scaling; $v$ : drift term; $\sigma$ : noise standard deviation. (C) The Bin Gaze model replicated the main behavioural relationships in a similar way to the original continuous gaze model. The four panels present four relevant behavioural relationships comparing model predictions and overall participant behaviour: (top left) responses time were faster (shorter RT) when the choice was easier (i.e. bigger differences in the number of dots between the patches); (top right) probability of choosing the left patch increased when the number of dots was higher in the patch on the left side ( $\Delta$ Dots = DotsLeft - Dots Right); (bottom left) the probability of choosing an alternative depended on the gaze difference ( $\Delta$ Gaze $=g_{\text {Left }}-\mathrm{g}_{\text {Right }}$ ); and (bottom right) the probability of choosing an item that was fixated longer than the other, corrected by the actual evidence $(\Delta$ Dots), depicted a residual effect of gaze on choice. Solid dots depict the mean of the data across participants. Lighter dots present the mean value for each participant across bins. Solid grey lines show the average for model simulations. Data are binned for visualization. ${ }^{* *}$ : $<<0.01$.

A

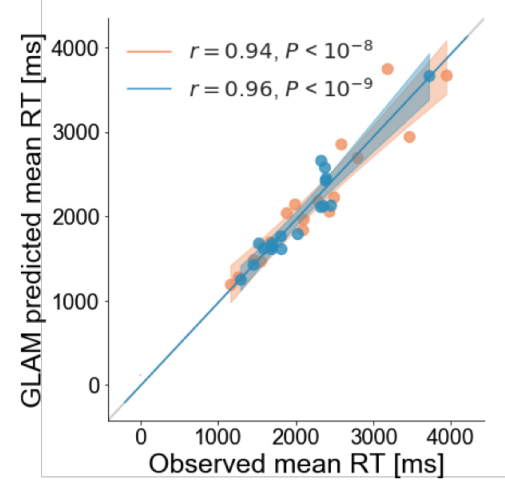

B

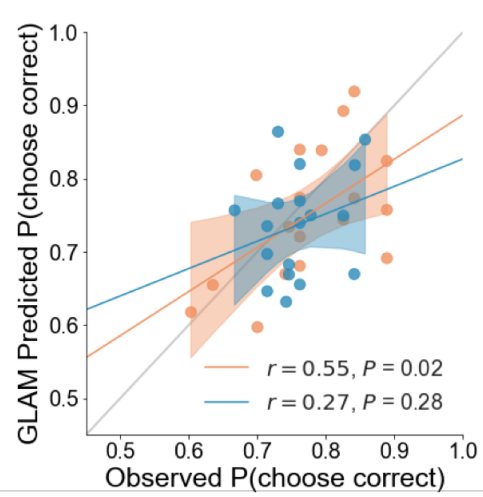

C

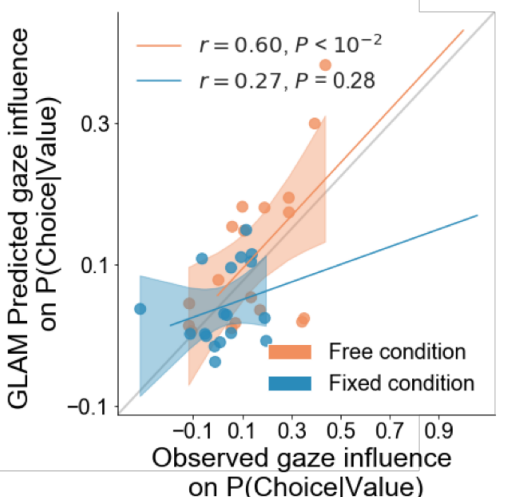

Figure S5.2. Individual out-of-sample GLAM predictions for behavioural measures in free and fixed sampling conditions. The correlations between observed data and predictions of the model for individual (A) mean RT, (B) the probability of choosing the correct patch, and (C) the gaze influence in choice probability are presented. In the fixed sampling condition, the correlation between the performance (probability of correct) of individual participants and the model predictions was found not statistically significant, indicating the model was not completely accurate in predicting participant-level performance. However, the model captures group-level performance (as depicted in Figure 5B), since predicted trials had higher than chance accuracy and a similar range of performance as observed trials (accuracy is between 0.6-0.9 for observed and predicted). Regarding the gaze influence measure (residual effect of gaze on choice, once the effect of evidence is accounted for), the free sampling model predicts this effect significantly at the participant level, but the fixed sampling model did not. Since in the fixed sampling model, in practical terms there is no gaze bias $(\gamma \approx 1)$, we expected the model would have trouble predicting any residual gaze influence. Dots depict the average of observed and predicted measures for each participant. In the free sampling condition the model prediction correlated significantly with observed accuracy and gaze influence, at the participant-level. Lines depict the slope of the correlation between observations and predictions. Dots indicate the average measure for each participant's observed and predicted data. Mean 95\% confidence intervals are represented by the shadowed region. All model predictions are simulated using parameters estimated from individual fits for even-numbered trials. 


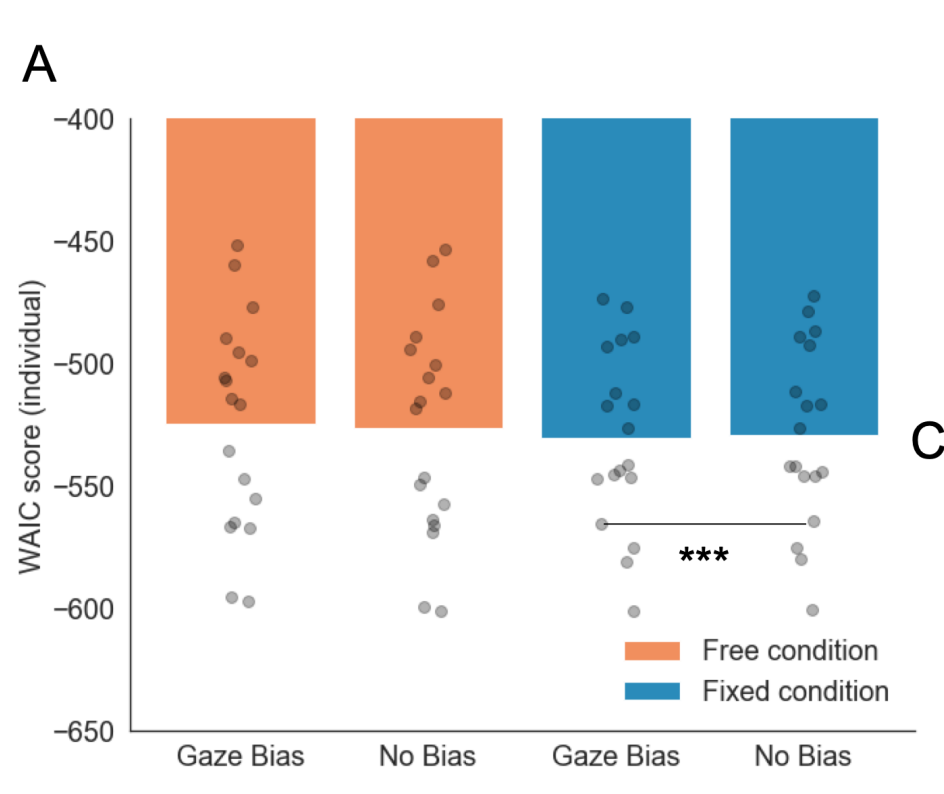

B
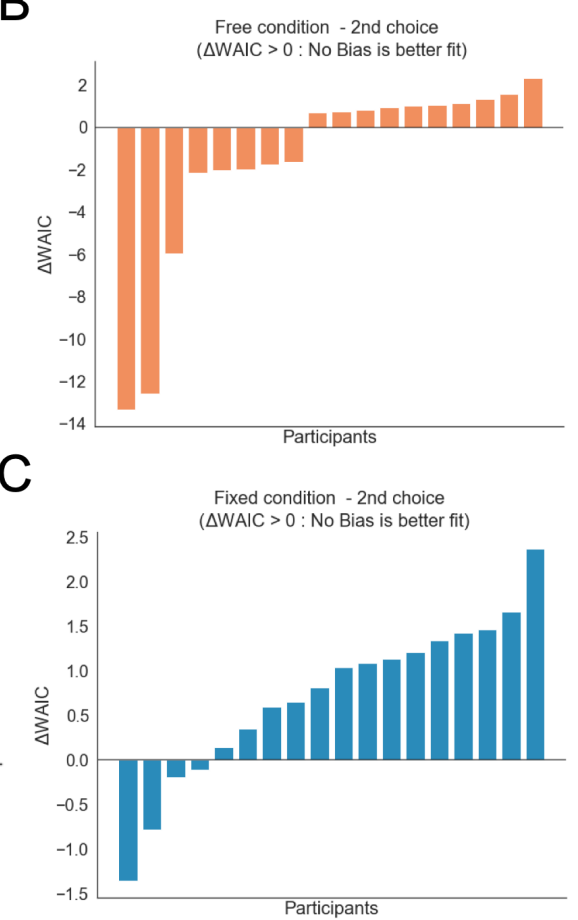

Figure S5.3. GLAM model comparison for free and fixed sampling conditions. (A) WAIC scores for free and fixed sampling models did not report significant difference in fit between the conditions (mean WAIC $_{\text {Free }}=-524.58$; mean WAIC Fixed $=-530.004 ; \mathrm{t}_{17}=0.98, \mathrm{p}=0.34, \mathrm{~ns}$ ). As an additional check, we fitted new models for both conditions without the gaze bias (no bias, $\gamma=1$ ). We found that in the fixed gaze condition, the no bias model was the most parsimonious model (Mean WAIC Fixed/GazeBias $=-530.004$; Mean WAIC $\left._{\text {Fixed } / \text { NoBias }}=-529.29 ; \mathrm{t}_{17}=3.304, \mathrm{p}<0.01\right)$. No differences were found between the gaze bias and no bias models in the free sampling condition (Mean WAIC Free/GazeBias $=-524.58$; Mean WAIC Free $/$ NoBias $=-526.23$; $\left.\mathrm{t}_{17}=1.537, \mathrm{p}=0.14, \mathrm{~ns}\right)$. (B-C) Differences in WAIC score between Gaze Bias - No Bias ( $\triangle$ WAIC) models were calculated for each individual participant and experimental condition. This corroborated that the No Bias model has a better fit in the fixed sampling condition only (C). WAIC scores are presented using log scale. A higher log-score indicates a model with better predictive accuracy. ${ }^{* * *}$ : $<<0.001$. 
bioRxiv preprint doi: https://doi.org/10.1101/2021.06.29.450332; this version posted June 30, 2021. The copyright holder for this preprint (which was not certified by peer review) is the author/funder, who has granted bioRxiv a license to display the preprint in perpetuity. It is made available under aCC-BY-NC-ND 4.0 International license.
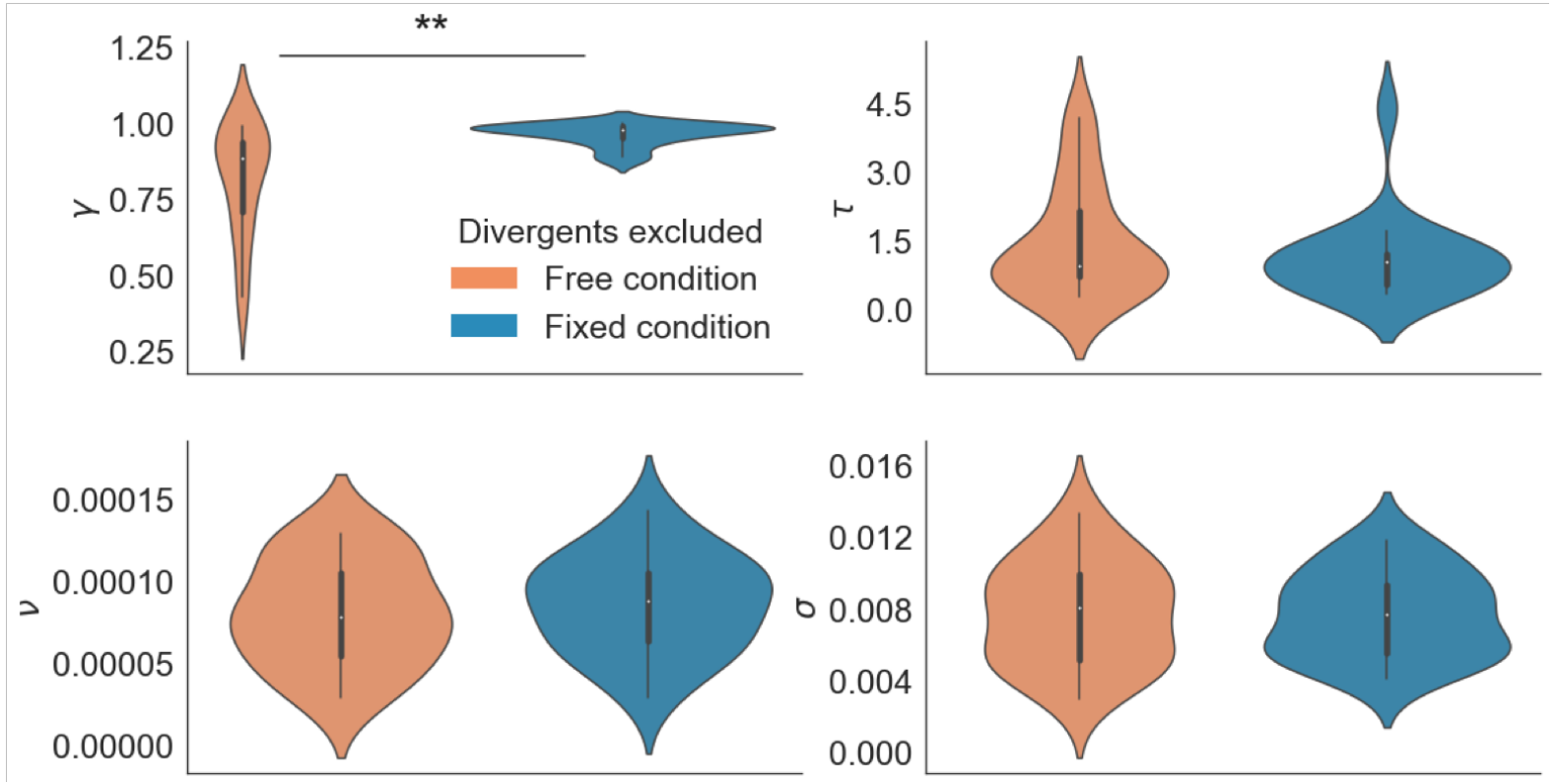

Figure S5.4. GLAM parameters for free and fixed sampling conditions. Participants for which parameter estimation did not converge were removed from the analysis (4 participants). The results reported in the main text were still observed: a higher gaze bias (lower $\gamma$ parameter) in the free sampling condition (Mean $\gamma_{\text {Free }}=0.786$, Mean $\gamma_{\text {Fixed }}=0.961, \mathrm{t}_{17}=-3.033 ; \mathrm{p}<0.01$ ). No significant differences were found for the other parameters. $\gamma$ : gaze bias; $\tau$ : evidence scaling; $v$ : drift term; $\sigma$ : noise standard deviation. ${ }^{* *}$ : $\mathrm{p}<0.01$ 


\section{S6: A Descriptive Model of Confirmatory Information Processing}

1063

1064

1065

1066

1067

1068

1069

1070

1071

1072

1073

1074

1075

1076

1077

1078

1079

1080

1081

1082

1083

1084

1085

1086

1087

1088

1089

1090

1091

1092

1093

1094

1095

1096

1097

1098

1099

1100

1101

1102

1103

1104

1105

1106

1107

1108

We have designed the following descriptive economic decision-making model that can be used to capture the findings described above. There is a set of states of the world that denote the number of dots in each part of the screen, denoted $\left(\omega^{L}, \omega^{R}\right) \in \mathbb{R}_{+}^{2}$. In what follows, for any probability measure $\mu$ over $\mathbb{R}_{+}^{2}$, denote by $\mu\left(\omega^{L}>\omega^{R}\right)$ the probability that $\mu$ assigns to the event that $\omega^{L}$ is above $\omega^{R}$; and by $B U(\mu, A)$ the Bayesian update of $\mu$ using information $A$. Subjects start with a symmetric prior $\mu_{0}$.

First Stage: Subject go through a sampling phase in which they gather information about the number of dots in each screen. They get two noisy signals about each component of the state, $x_{1}^{L}$ and $x_{1}^{R}$, for which for simplicity we assume normally distributed noise:

$$
\begin{aligned}
& x_{1}^{L}=\omega^{L}+\epsilon_{1}^{L} \\
& x_{1}^{R}=\omega^{R}+\epsilon_{1}^{R}
\end{aligned}
$$

where $\epsilon_{1}^{L} \sim N\left(0, \sigma_{1}^{L}\right), \epsilon_{1}^{R} \sim N\left(0, \sigma_{1}^{R}\right)$. For generality, we may allow the variances to be different, in case individuals follow an adaptive search procedure that leads to asymmetric information acquisition. For simplicity, we assume here that they are the same, $\sigma_{1}^{L}=\sigma_{1}^{R}=\sigma^{2}$.

At the end of the information gathering stage, subjects form a posterior about the state of the world, $\widehat{\mu_{1}}=B U\left(\mu_{0} \mid x_{1}^{R}, x_{1}^{L}\right)$. They are then asked to choose an action $a_{1} \in\{L, R\}$ and a confidence level $c$. Following standard arguments, they choose $a_{1}=L$ if $\widehat{\mu_{1}}\left(\omega^{L}>\omega^{R}\right)>$ $0.5, a_{1}=R$ if $\widehat{\mu_{1}}\left(\omega^{L}>\omega^{R}\right)<0.5$, and randomize with equal probability between $L$ and $R$ if $\widehat{\mu_{1}}\left(\omega^{L}>\omega^{R}\right)=0.5$ (a probability 0 event).

They report a confidence

$$
c=2 *\left|\widehat{\mu_{1}}\left(\omega^{L}>\omega^{R}\right)-\frac{1}{2}\right| .
$$

Note that $c=1$ if $\widehat{\mu_{1}}\left(\omega^{L}>\omega^{R}\right) \in\{1,0\}, c=0$ if $\widehat{\mu_{1}}\left(\omega^{L}>\omega^{R}\right)=0.5$.

Second Stage: The key departure from the standard normative model of decision-making is that the beliefs that individuals have at the beginning of stage 2 are not the posteriors they obtained at the end of stage 1 , but are also influenced by their choice. Denote ${\widehat{\mu_{1}}}^{D}$ the belief over the state of the world that the individual would obtain if she only observed her choice - given the decision rule above - but did not take into account the signals $x_{1}^{L}$ and $x_{1}^{R}$, i.e., ${\widehat{\mu_{1}}}^{D}=B U\left(\mu_{0}, a_{1}\right)$. We posit that the belief used by the individual at the beginning of the second stage, $\mu_{2}$, is a convex combination of this belief and the posterior at the end of period 1, i.e.,

$$
\mu_{2}=\theta{\widehat{\mu_{1}}}^{D}+(1-\theta) \widehat{\mu_{1}}
$$

where $\theta \in[0,1]$ indicates the degree of distortion: when $\theta=0$ beliefs are not distorted; when $\theta=1$, choices in the second stage only use the more limited information contained in the choice of stage 1 , and not the full information coming from observed signals. The use of the linear structure is not relevant but simplifies the analysis. 
1109

1110

1111

1112

1113

1114

1115

1116

1117

1118

1119

1120

1121

1122

1123

1124

1125

1126

1127

1128

1129

1130

1131

1132

1133

1134

1135

1136

1137

1138

1139

1140

1141

1142

1143

1144

1145

1146

1147

1148

1149

1150

1151
In the second stage, subjects have to decide the sampling strategy. We posit that the fraction of time spent on Left is determined (with noise) as a strictly increasing function of the belief $\mu_{2}$ : subjects spend more time on average on the area where they believe are more dots. In Sepulveda et al. (2020) a model is described that generates a related tendency. In particular, we posit that the fraction of time spent on the left circle, $s$, is determined by a standard logistic function

$$
s=\frac{1}{1+e^{-k\left(\mu_{2}\left(\omega^{L}>\omega^{R}\right)-0.5\right)}}
$$

Through this sampling, individuals receive signals

$$
\begin{aligned}
& x_{2}^{L}=\omega^{L}+\epsilon_{2}^{L} \\
& x_{2}^{R}=\omega^{R}+\epsilon_{2}^{R}
\end{aligned}
$$

where $\left.\epsilon_{1}^{L} \sim N\left(0, \frac{\sigma^{2}}{s}\right)\right), \epsilon_{1}^{R} \sim N\left(0, \frac{\sigma^{2}}{1-s}\right)$, so that better information is obtained about each dimension the more time spent contemplating it. Using this, subjects form a posterior $\widehat{\mu_{2}}=B U\left(\mu_{2}, \mid x_{2}^{L}, x_{2}^{R}\right)$.

Finally, subjects choose an action $a_{2} \in\{L, R\}$ and a confidence level $c$. Following standard arguments, they choose $a_{1}=L$ if $\widehat{\mu_{2}}\left(\omega^{L}>\omega^{R}\right)>0.5, a_{1}=R$ if $\widehat{\mu_{2}}\left(\omega^{L}>\omega^{R}\right)<0.5$, and randomize with equal probability between $L$ and $R$ if $\widehat{\mu_{2}}\left(\omega^{L}>\omega^{R}\right)=0.5$ (again, a 0 probability event).

This model is reminiscent of the Second-Order Model of Fleming and Daw (2017), but with the difference that we are not introducing a choice-affecting-beliefs component at the time of reporting confidence, but only at the beginning of the second stage.

This model implies that, on average, subjects will sample more from the patch they had previously chosen; and that this effect is stronger the higher their confidence in their choice-which is what we see in the data.

When $\theta>0$, the previous choice affects the sampling strategy even controlling for confidence-which is also what we find. Whenever ${\widehat{\mu_{1}}}^{D}\left(\omega^{L}>\omega^{R}\right)>\widehat{\mu_{1}}\left(\omega^{L}>\omega^{R}\right)>$ 0.5 , the presence of $\theta>0$ will lead to $\mu_{2}\left(\omega^{L}>\omega^{R}\right)>\widehat{\mu_{1}}\left(\omega^{L}>\omega^{R}\right)>0.5$. This, in turn, will distort her later sampling strategy as well as her future beliefs: the individual will require even stronger information to change her mind. The opposite happens when $\widehat{\mu_{1}}\left(\omega^{L}>\omega^{R}\right)>{\widehat{\mu_{1}}}^{D}\left(\omega^{L}>\omega^{R}\right)>0.5$ : in this case $\widehat{\mu_{1}}\left(\omega^{L}>\omega^{R}\right)>\mu_{2}\left(\omega^{L}>\omega^{R}\right)>0.5$, which implies that the individual acts as if she were less confident of her choice. Previous choice will affect the sampling strategy, but now less so. 
1152

1153

1154

1155

1156

1157

1158

1159

1160

1161

1162

1163

1164

1165

1166

\section{S7: Model Comparisons}

\section{Sampling Time Models}

To investigate the effects of choice, dot difference, confidence, and response time on sampling we compared 6 hierarchical regression models. These models are presented below. The BIC scores for each model in each experiment are plotted in Figure S7.1. The best fitting models according to BIC-scores were Model 5 in experiment 1 and Model 3 in experiment 2 . We chose to present Model 5 for both experiments as we were interested in the contribution of confidence to biased sampling.

\section{Sampling Time Models}

\section{Models Formula}

$1 \quad$ Sampling Time Difference $\sim \mathcal{N}\left(\beta_{0}+\beta_{1}[\right.$ Choice $\left.]+\varepsilon\right)$

$2 \quad$ Sampling Time Difference $\sim \mathcal{N}\left(\beta_{0}+\beta_{1}[\right.$ Dot Difference] $+\varepsilon)$

$3 \quad$ Sampling Time Difference $\sim \mathcal{N}\left(\beta_{0}+\beta_{1}[\right.$ Choice $]+\beta_{2}[$ Dot Difference $\left.]+\varepsilon\right)$

$4 \quad$ Sampling Time Difference $\sim \mathcal{N}\left(\beta_{0}+\beta_{1}\right.$ [Choice] $+\beta_{2}$ [Confidence] + $\beta_{3}[$ Choice * Confidence $]+\varepsilon$ )

$5 \quad$ Sampling Time Difference $\sim \mathcal{N}\left(\beta_{0}+\beta_{1}[\right.$ Choice $]+\beta_{2}[$ Dot Difference] + $\beta_{3}[$ Confidence $]+\beta_{4}[$ Choice $*$ Confidence $]+\varepsilon$ ]

6 Sampling Time Difference $\sim \mathcal{N}\left(\beta_{0}+\beta_{1}[\right.$ Choice $]+\beta_{2}[$ Dot Difference $]+$ $\beta_{3}[$ Confidence $]+\beta_{4}[$ Reaction Time $]+\beta_{5}[$ Choice $*$ Confidence $]+\beta_{6}[$ Choice $*$ Reaction Time $]+\varepsilon$ ) 

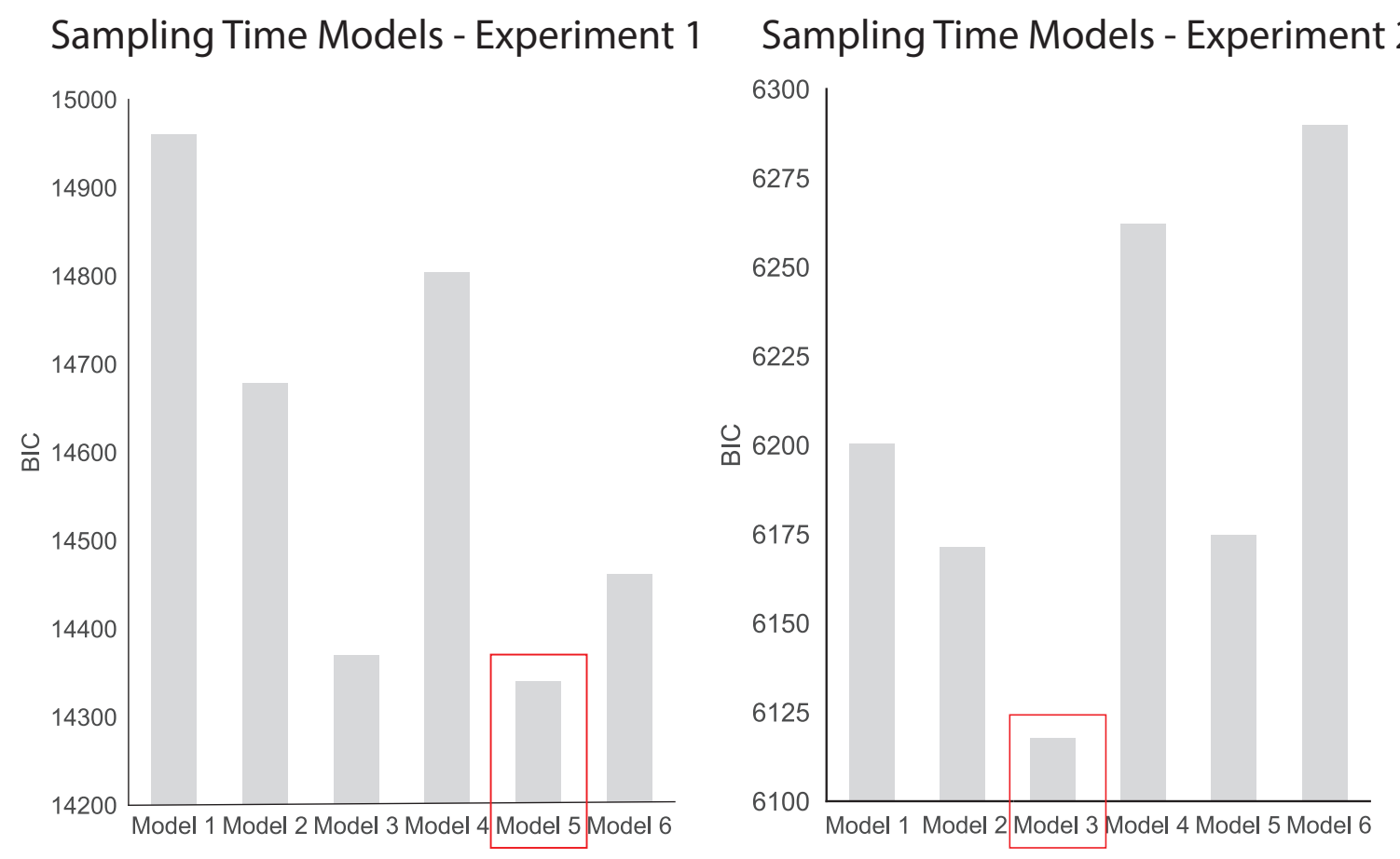

Figure S7.1. BIC comparison of the sampling time models for experiments 1 and 2.

Model 5 fit the data from experiment 1 the best $(B I C=14340.8$ ), whereas Model 3 was the best fit for the data in experiment $2(\mathrm{BIC}=6117.8)$.

\section{Change of Mind Models}

1174 To investigate the effects of dot difference, sampling, confidence, and response time on change of mind we compared 5 hierarchical regression models. These models are presented below. The BIC scores for each model in each experiment are plotted in Figure S7.2. Model 5 includes a dummy variable coding whether or not the trial was in the control condition or not (in which sampling was fixed). As such, this model is only applicable to experiment 2 . The best fitting models according to BIC-scores were Model 3 in experiment 1 and Model 5 in experiment 2 . 


\section{Change of Mind Models}

1184

\begin{tabular}{|c|c|}
\hline Models & Formula \\
\hline 1 & Change of Mind $\sim \operatorname{logit}^{-1}\left(\beta_{0}+\beta_{1}[\right.$ Dot Difference $\left.]+\varepsilon\right)$ \\
\hline 2 & Change of Mind $\sim \operatorname{logit}^{-1}\left(\beta_{0}+\beta_{1}[\right.$ Dot Difference $]+\beta_{2}[$ Sampling Bias $\left.]+\varepsilon\right)$ \\
\hline 3 & $\begin{array}{l}\text { Change of Mind } \sim \operatorname{logit}^{-1}\left(\beta_{0}+\beta_{1}[\text { Dot Difference }]+\beta_{2}[\text { Sampling Bias }]+\right. \\
\left.\beta_{3}[\text { Confidence }]+\varepsilon\right)\end{array}$ \\
\hline 4 & $\begin{array}{l}\text { Change of Mind } \sim \operatorname{logit}^{-1}\left(\beta_{0}+\beta_{1}[\text { Dot Difference }]+\beta_{2}[\text { Sampling Bias }]+\right. \\
\left.\beta_{3}[\text { Confidence }]+\beta_{4}[\text { Reaction Time }]+\varepsilon\right)\end{array}$ \\
\hline 5 & $\begin{array}{l}\text { Change of Mind } \sim \operatorname{logit}^{-1}\left(\beta_{0}+\beta_{1}[\text { Dot Difference }]+\beta_{2}[\text { Sampling Bias }]+\right. \\
\beta_{3}[\text { Confidence }]+\beta_{4}[\text { Fixed Sampling }]+\beta_{5}[\text { Sampling Bias * Fixed Sampling }] \\
+\varepsilon)\end{array}$ \\
\hline
\end{tabular}
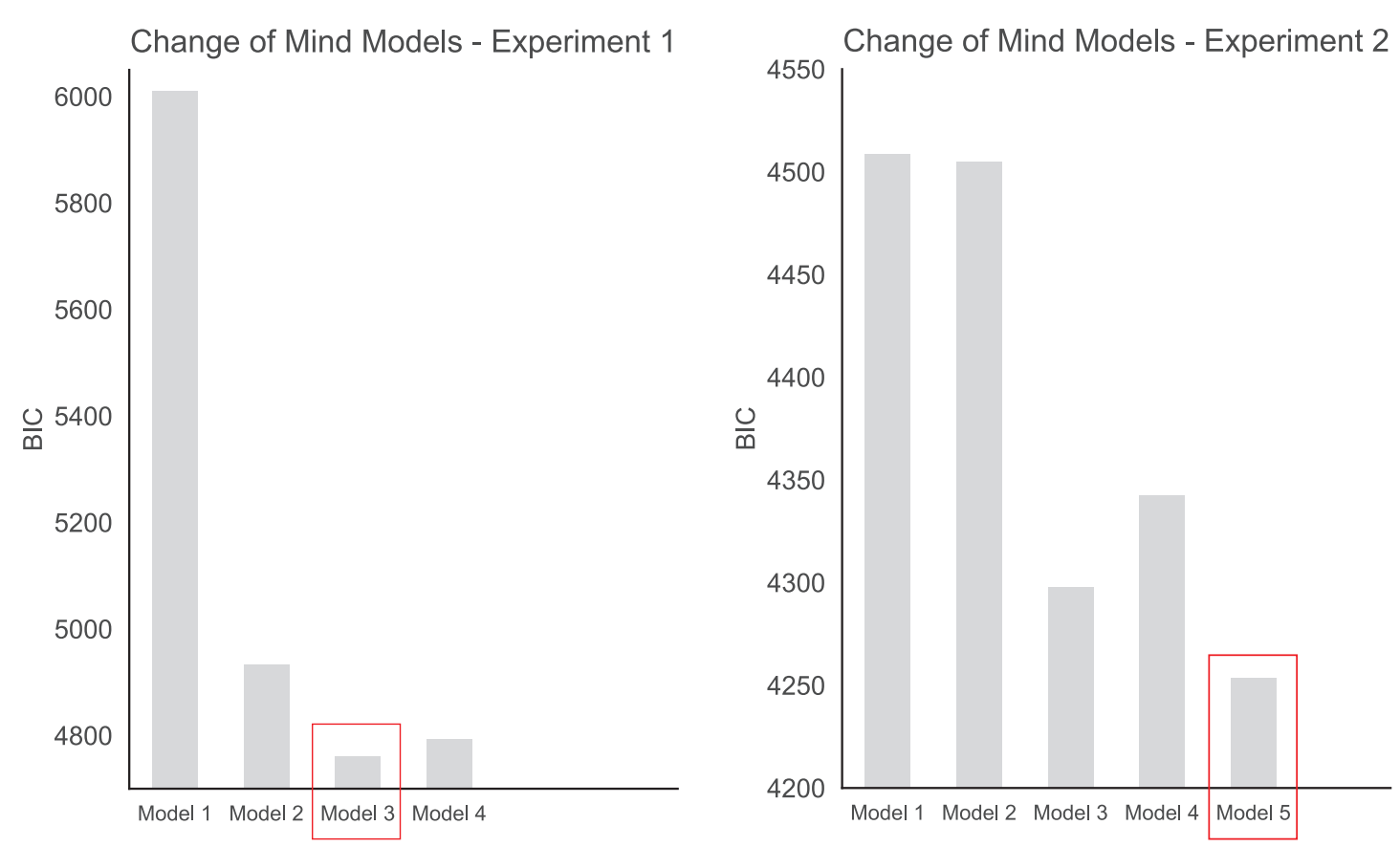

Figure S7.2. BIC comparison of the change of mind models for experiments 1 and 2. data in experiment 2 (BIC $=4253.7)$. 